\title{
Enhanced UV radiation and dense clumps in the molecular outflow of Mrk 231^
}

\author{
Claudia Cicone ${ }^{1,2}$, Roberto Maiolino ${ }^{3,4}$, Susanne Aalto ${ }^{5}$, Sebastien Muller ${ }^{5}$, and Chiara Feruglio ${ }^{6}$ \\ ${ }^{1}$ Institute of Theoretical Astrophysics, University of Oslo, PO Box 1029, Blindern 0315, Oslo, Norway \\ e-mail: claudia.cicone@astro.uio.no \\ 2 INAF-Osservatorio Astronomico di Brera, Via Brera 28, 20121 Milan, Italy \\ 3 Cavendish Laboratory, University of Cambridge, 19 J. J. Thomson Ave, Cambridge CB3 0HE, UK \\ ${ }_{5}^{4}$ Kavli Institute for Cosmology, University of Cambridge, Madingley Road, Cambridge CB3 0HA, UK \\ 5 Department of Space, Earth and Environment, Chalmers University of Technology, Onsala Observatory, 43992 Onsala, Sweden \\ ${ }^{6}$ INAF - Osservatorio Astronomico di Trieste, Via G.B. Tiepolo 11, 34143 Trieste, Italy
}

Received 27 September 2019 / Accepted 25 November 2019

\section{ABSTRACT}

\begin{abstract}
We present interferometric observations of the $\mathrm{CN}(1-0)$ line emission in Mrk 231 and combine them with previous observations of $\mathrm{CO}$ and other $\mathrm{H}_{2}$ gas tracers to study the physical properties of the massive molecular outflow. We find a strong boost of the $\mathrm{CN} / \mathrm{CO}$ (1-0) line luminosity ratio in the outflow of Mrk 231, which is unprecedented compared to any other known Galactic or extragalactic astronomical source. For the dense gas phase in the outflow traced by the $\mathrm{HCN}$ and CN emissions, we infer $X_{\mathrm{CN}} \equiv[\mathrm{CN}] /\left[\mathrm{H}_{2}\right]>X_{\mathrm{HCN}}$ by at least a factor of three, with $\mathrm{H}_{2}$ gas densities of $n_{\mathrm{H}_{2}} \sim 10^{5-6} \mathrm{~cm}^{-3}$. In addition, we resolve for the first time narrow spectral features in the $\mathrm{HCN}(1-0)$ and $\mathrm{HCO}^{+}(1-0)$ high-velocity line wings tracing the dense phase of the outflow. The velocity dispersions of these spectral features, $\sigma_{\mathrm{v}} \sim 7-20 \mathrm{~km} \mathrm{~s}^{-1}$, are consistent with those of massive extragalactic giant molecular clouds detected in nearby starburst nuclei. The $\mathrm{H}_{2}$ gas masses inferred from the $\mathrm{HCN}$ data are quite high, $M_{\text {mol }} \sim 0.3-5 \times 10^{8} M_{\odot}$. Our results suggest that massive complexes of denser molecular gas survive embedded into the more diffuse $\mathrm{H}_{2}$ phase of the outflow, and that the chemistry of these outflowing dense clouds is strongly affected by UV radiation.
\end{abstract}

Key words. galaxies: active - galaxies: evolution - galaxies: individual: Mrk 231 - galaxies: interactions - submillimeter: galaxies galaxies: ISM

\section{Introduction}

Mrk 231 is a late-stage merger, classified as an ultra-luminous infrared galaxy (ULIRG) $\left(L_{\mathrm{IR}}(8-1000 \mu \mathrm{m})=1.3 \times 10^{46} \mathrm{erg} \mathrm{s}^{-1}\right)$, which probably is on its path to becoming an elliptical galaxy (Lípari et al. 2005). At least $\sim 30 \%$ of the IR luminosity is due to dust heated by an active galactic nucleus (AGN; Nardini et al. (2010), but Veilleux et al. (2009) estimated a higher AGN contribution). Despite its weak emission in the hard X-ray band (Teng et al. 2014; Piconcelli et al. 2013; Braito et al. 2004), the high AGN bolometric luminosity $\left(L_{\mathrm{AGN}} \sim 8 \times 10^{45} \mathrm{erg} \mathrm{s}^{-1}\right.$, Leighly et al. 2014) makes Mrk 231 the closest quasar known. However, most of the IR luminosity traces a young starburst (age < 250 Myr, Davies et al. 2007) with a star formation rate (SFR) of SFR $\gtrsim 200 M_{\odot} \mathrm{yr}^{-1}$ (Taylor et al. 1999), fed by $\gtrsim 10^{9} M_{\odot}$ of molecular $\left(\mathrm{H}_{2}\right)$ gas distributed in a kiloparsec-size disc (Downes \& Solomon 1998).

The multi-wavelength and multi-scale emission of Mrk 231 is overwhelmingly affected by explosive phenomena, which are a manifestation of feedback from the star formation and quasar activity, as predicted by theoretical models (Chevalier \& Clegg 1985; Silk \& Rees 1998; Murray et al. 2005; Faucher-Giguère \& Quataert 2012; Zubovas \& King 2012, 2014; Nims et al. 2015; Thompson et al. 2016; Ishibashi et al.2018) and observed in hydrodynamical simulations (Di Matteo et al. 2005;

\footnotetext{
$\star$ The reduced datacubes are only available at the CDS via anonymous ftp to cdsarc.u-strasbg.fr $(130.79 .128 .5)$ or via http: //cdsarc.u-strasbg.fr/viz-bin/cat/J/A+A/633/A163
}

Sijacki et al. 2007; Costa et al. 2015, 2018; Biernacki \& Teyssier 2018; Richings \& Faucher-Giguère 2018; Schneider et al. 2018). On nuclear scales, Feruglio et al. (2015) reported observations of a possible X-ray ultra-fast wind with $v=20000 \mathrm{~km} \mathrm{~s}^{-1}$. Broad absorption lines (BALs) were detected in both low- and high-ionisation species, classifying Mrk 231 as an iron lowionization broad absorption-line (FeLoBAL) quasar (Lípari et al. 2009). The high-velocity material responsible for the BALs is believed to dominate even the Ly $\alpha$, CIV, and CIII] emission lines, which all show spectral profiles blueshifted by $-3500 \mathrm{~km} \mathrm{~s}^{-1}$ (Veilleux et al. 2013). In addition to the BAL winds, which are confined within $r<10 \mathrm{pc}$, Mrk 231 hosts one of the best-studied galaxy-scale outflows that was revealed for the first time in $\mathrm{OH}$ absorption by Fischer et al. (2010) and in CO emission by Feruglio et al. (2010). Based on these earlier observations, it was estimated that the outflow entrains $\sim 3 \times 10^{8} M_{\odot}$ of $\mathrm{H}_{2}$ gas and extends out to at least $r \sim 1 \mathrm{kpc}$ into the interstellar medium (ISM; Cicone et al. 2012). Blueshifted NaID (Rupke \& Veilleux 2011; Lípari et al. 2009) and H I $21 \mathrm{~cm}$ (Morganti et al. 2016; Teng et al. 2013) absorption features have also been detected, and they trace the H I gas component of the outflow.

At present, most galaxy-scale molecular outflows have been studied exclusively through low- $J$ CO transitions (Cicone et al. 2014; Pereira-Santaella et al. 2018; Fluetsch et al. 2019). Because of their low critical density and of the high abundance of CO in the ISM (if CO is sufficiently shielded), these lines are ubiquitous tracers of $\mathrm{H}_{2}$ gas, including the diffuse envelopes of molecular clouds that do not host active star 
formation. On the other hand, transitions from higher critical density species such as $\mathrm{HCN}, \mathrm{HCO}^{+}$, and $\mathrm{HNC}$ are expected to arise mainly from the denser regions of molecular complexes that are associated with star formation sites (Gao \& Solomon 2004). Therefore, these molecules (including CN, see below) are commonly referred to as "dense gas" tracers, and we adopt this terminology throughout the text. However, we note that a significant portion of the emission from such tracers may still be associated with gas whose density is far lower than the nominal critical density $\left(n_{\text {crit }}\right)$ of the corresponding molecular transition. $n_{\text {crit }}$ is indeed calculated analytically using the optically thin approximation, and therefore it does not account for radiative trapping (Scoville \& Solomon 1974), which can lower the volume density that is required to excite the line by more than one order of magnitude with respect to $n_{\text {crit }}$. To adequately consider line trapping, Shirley (2015) proposed using the effective excitation density $\left(n_{\text {eff }}\right)$, which is empirically defined as the gas density that produces a detectable integrated line flux arbitrarily set equal to $\int T_{\mathrm{R}} \mathrm{d} v=1 \mathrm{~K} \mathrm{~km} \mathrm{~s}^{-1}$, where $T_{\mathrm{R}}$ is the radiation temperature. We refer to Shirley (2015) for further details on the $n_{\mathrm{eff}}$ computation, but we note that there are different definitions of $n_{\text {eff }}$ in the literature, see also Leroy et al. (2017). Furthermore, the $n_{\text {crit }}$ computation in general assumes molecular hydrogen $\left(\mathrm{H}_{2}\right)$ as the only collisional partner and thus neglects collisions with electrons and atomic hydrogen. Collisions with electrons may become relevant in a medium where a large portion of carbon is ionised but hydrogen is in the molecular phase, leading to the excitation of molecules such as $\mathrm{HCN}$ even in lower density $\mathrm{H}_{2}$ gas $\left(n_{\mathrm{H}_{2}} \sim 10^{3} \mathrm{~cm}^{-3}\right.$, e.g. Goldsmith \& Kauffmann 2017 ; Kauffmann et al. 2017).

The first detection of $\mathrm{HCN}, \mathrm{HCO}^{+}$, and $\mathrm{HNC}$ in the outflow of Mrk 231 was reported by Aalto et al. (2012). This outflow has so far been studied in eight different species, four detected in absorption by Herschel (e.g. $\mathrm{OH},{ }^{18} \mathrm{OH}, \mathrm{H}_{2} \mathrm{O}$, and $\mathrm{OH}^{+}$, Fischer et al. 2010; Sturm et al. 2011; González-Alfonso et al. 2014, 2018), and four imaged in emission using (sub-) millimetre interferometry (e.g. $\mathrm{CO}, \mathrm{HCN}, \mathrm{HNC}$, and $\mathrm{HCO}^{+}$, see Feruglio et al. 2010, 2015; Cicone et al. 2012; Aalto et al. 2012, 2015; Alatalo 2015; Lindberg et al. 2016).

We here report the detection of exceptionally bright cyanide radical $(\mathrm{CN}) N=1-0$ emission arising from the outflow of Mrk 231. CN is a tracer of high-density molecular gas (for $T_{k}=100 \rightarrow 10 \mathrm{~K}, n_{\text {crit }} \simeq 6.4 \times 10^{4} \rightarrow 4.1 \times 10^{5} \mathrm{~cm}^{-3}$ and $n_{\mathrm{eff}} \simeq 4.6 \times 10^{3} \rightarrow 3.8 \times 10^{4} \mathrm{~cm}^{-3}$, assuming a reference column density of $N_{\mathrm{CN}}=10^{14} \mathrm{~cm}^{-2}$, Shirley (2015)). The production of $\mathrm{CN}$ is aided by the presence of moderate $\mathrm{UV}$ fields, either through the reaction $\mathrm{CH}+\mathrm{N} \rightarrow \mathrm{CN}+\mathrm{H}$ because $\mathrm{CH}$ is formed rapidly in a medium where both $\mathrm{C}^{+}$ and $\mathrm{H}_{2}$ are abundant (Fuente et al. 1993; Meier et al. 2015), or through the photodissociation of $\mathrm{HCN}: \mathrm{HCN}+\gamma_{\mathrm{UV}} \rightarrow \mathrm{CN}+\mathrm{H}$ (Boger \& Sternberg 2005). For these reasons, CN is expected to be more abundant in photon-dominated regions (PDRs), which are portions of the clouds where the chemistry is dominated by the interactions with UV photons (e.g. Aalto et al. 2002; Rodriguez-Franco et al. 1998), but possibly also in the presence of X-rays and/or cosmic rays (Meijerink et al. 2007; García-Burillo et al. 2010; Harada et al. 2015). Observations of Galactic star-forming regions have shown that the mere presence of dense molecular gas is not itself a prior for higher $\mathrm{CN}$ emission. Instead, CN tends to be brighter on the surfaces of molecular clouds that are exposed to interstellar UV radiation (PDRs), but also inside molecular clouds that host young massive stars (Yamagishi et al. 2018). Notably, CN remains very sensitive to variations in star formation activity even when its emission is averaged across kiloparsec-scale regions in extragalactic sources (Watanabe et al. 2014).

We here also present the first detection of spectrally resolved features in the broad wings of the $\mathrm{HCN}(1-0)$ and $\mathrm{HCO}^{+}(1-0)$ emission lines. Their velocity dispersions are consistent with individual massive molecular clouds entrained in the outflow. We discuss their properties and compare them with known Galactic and extragalactic giant molecular clouds (GMCs).

The paper is organised as follows. The observations are described in Sect. 2. In Sect. 3.1 we show the $\mathrm{CN}(1-0)$ data and combine them with previous (sub-)millimetre observations of other molecular lines to study the physical properties of the outflowing $\mathrm{H}_{2}$ gas. In Sect. 3.2 we present and discuss new observational evidence for an intrinsic clumpiness of the broad spectral wings of the $\mathrm{HCN}(1-0)$ and $\mathrm{HCO}^{+}(1-0)$ lines. Our results are discussed in Sect. 4 and summarised in Sect. 5. Throughout the paper we adopt a standard $\Lambda$ CDM cosmological model with $H_{0}=67.8 \mathrm{~km} \mathrm{~s}^{-1} \mathrm{Mpc}^{-1}, \Omega_{\Lambda}=0.692, \Omega_{\mathrm{M}}=0.308$ (Planck Collaboration XIII 2016). At the distance of Mrk 231 (redshift $z=0.04217$, luminosity distance $D_{\mathrm{L}}=192.4 \mathrm{Mpc}$ ), the physical scale is $0.859 \mathrm{kpc} \operatorname{arcsec}^{-1}$. Uncertainties correspond to $1 \sigma$ statistical errors.

\section{Observations}

The $N=1-0 J=3 / 2-1 / 2$, and $J=1 / 2-1 / 2$ spin doublet transition of $\mathrm{CN}$ (hereafter, $\mathrm{CN}(1-0)$ ) consists of several hyperfine lines (five in the brighter $J=3 / 2-1 / 2$ group and four in the $J=1 / 2-1 / 2$ group, e.g. Skatrud et al. 1983), with a frequency separation that is much smaller than the molecular line width of Mrk 231 (maximum separation between the $J=3 / 2-1 / 2$ hyperfine levels, $\Delta v^{\max }=32.3 \mathrm{MHz}=85 \mathrm{~km} \mathrm{~s}^{-1}$ ). The $\mathrm{CN}$ (1-0) doublet was included within the $3.6 \mathrm{GHz}$ wide spectral bandwidth of the IRAM Plateau de Bure Interferometer (PdBI, now NOrthern Extended Millimeter Array) observations that also targeted the $\mathrm{CO}(1-0)$ transition and were described in Cicone et al. (2012). However, the $\mathrm{CN}(1-0)$ data are shown and analysed for the first time in this paper. The phase centre of these observations is $\operatorname{RA}(J 2000)=12: 56: 14.200$; $\operatorname{Dec}(\mathrm{J} 2000)=+56: 52: 25.0$. The other main technical parameters such as rest and observed frequency, date, array configuration, synthesised beam, and rms noise are summarised in Table 1. For data reduction and analysis we used the CLIC and MAPPING packages within the GILDAS software ${ }^{1}$. The $3 \mathrm{~mm}$ continuum adjacent to the $\mathrm{CO}(1-0)$ and $\mathrm{CN}(1-0)$ lines was estimated between $109.3037<v_{\text {obs }}[\mathrm{GHz}]<109.8779$ and $111.2470<v_{\mathrm{obs}}[\mathrm{GHz}]<111.8603$, and subtracted from the $u v$ visibility data prior to imaging and cleaning.

We here also use archival IRAM PdBI observations of other molecular gas tracers that have been detected in the outflow of Mrk 231 and were presented in previous publications. Technical details and relevant references for these archival datasets are also reported in Table 1.

\section{Results}

\section{1. $\mathrm{CN}$ and high-density tracers in the outflow}

The continuum-subtracted maps of the total $\mathrm{CN}(1-0)$ line emission and of the blue- and redshifted $\mathrm{CN}(1-0)$ components are presented in Fig. 1. For the line wings, we selected velocities that are not affected by blending of the two $\mathrm{CN}(1-0)$ spingroups. In addition to the well-known central concentration of $\mathrm{H}_{2}$ gas in

http://wWw.iram.fr/IRAMFR/GILDAS 
Table 1. IRAM PdBI observations of Mrk 231.

\begin{tabular}{|c|c|c|c|c|c|c|c|c|}
\hline $\begin{array}{l}\text { Line(s) } \\
\text { (1) }\end{array}$ & $\begin{array}{c}v_{\text {rest }} \\
{[\mathrm{GHz}]} \\
(2)\end{array}$ & $\begin{array}{c}v_{\mathrm{obs}} \\
{[\mathrm{GHz}]} \\
(3)\end{array}$ & $\begin{array}{l}\text { Date } \\
\text { (4) }\end{array}$ & $\begin{array}{l}\text { Conf. } \\
\text { (5) }\end{array}$ & $\begin{array}{c}\text { Clean beam } \\
{\left[{ }^{\prime \prime} x^{\prime \prime}\right]} \\
(6)\end{array}$ & 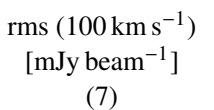 & $\begin{array}{l}\text { Ref. } \\
\text { (8) }\end{array}$ & Proj. ID \\
\hline $\mathrm{CN}(1-0)$ & $113.4910^{(\dagger)}$ & $108.8987^{(\dagger)}$ & 2010 & $6 \mathrm{C}$ & $2.8 \times 2.6$ & 0.47 & This work & UA26 \\
\hline $\mathrm{CO}(1-0)$ & 115.2712 & 110.6069 & 2009-2010 & $6 \mathrm{C} / 5 \mathrm{D}$ & $3.2 \times 2.8$ & 0.25 & Cicone et al. (2012) & UA26, TQ2F \\
\hline $\mathrm{CO}(2-1)$ & 230.5380 & 221.2096 & 2010 & $6 \mathrm{C} / 5 \mathrm{D}$ & $1.3 \times 1.0$ & 0.79 & Cicone et al. (2012) & UB26 \\
\hline $\mathrm{CO}(3-2)$ & 345.7960 & 331.8038 & 2012 & $6 \mathrm{D}$ & $1.5 \times 1.3$ & 2.1 & Feruglio et al. (2015) & V087 \\
\hline $\mathrm{HCN}(1-0)^{(\ddagger)}$ & 88.6319 & 85.0455 & 2011 & $6 \mathrm{~B}$ & $1.4 \times 1.2$ & 0.37 & Aalto et al. (2012) & U-D \\
\hline $\mathrm{HCN}(2-1)$ & 177.2610 & 170.0884 & 2012 & $5 \mathrm{D}$ & $2.9 \times 2.0$ & 0.60 & Lindberg et al. (2016) & W028 \\
\hline $\mathrm{HCN}(3-2)$ & 265.8862 & 255.1275 & 2012-2013 & $6 \mathrm{~A} / 6 \mathrm{~B}$ & $0.4 \times 0.3$ & 0.54 & Aalto et al. (2015) & V086, WA85 \\
\hline
\end{tabular}

Notes. (1) Molecular transition; (2) rest frequency; (3) observed (sky) frequency; (4) observing dates; (5) configuration of the interferometer; (6) synthesised beam size achieved after cleaning with natural weighting by using a support encompassing the $3 \sigma$ contour levels; (7) $1 \sigma$ rms per beam calculated in channels of $\Delta v=100 \mathrm{~km} \mathrm{~s}^{-1}$; (8) relevant references; (9) identification code of the observing programme. ${ }^{(\dagger)}$ Central frequency


and $\mathrm{HC}_{3} \mathrm{~N}(10-9)$ transitions (see Fig. 2).
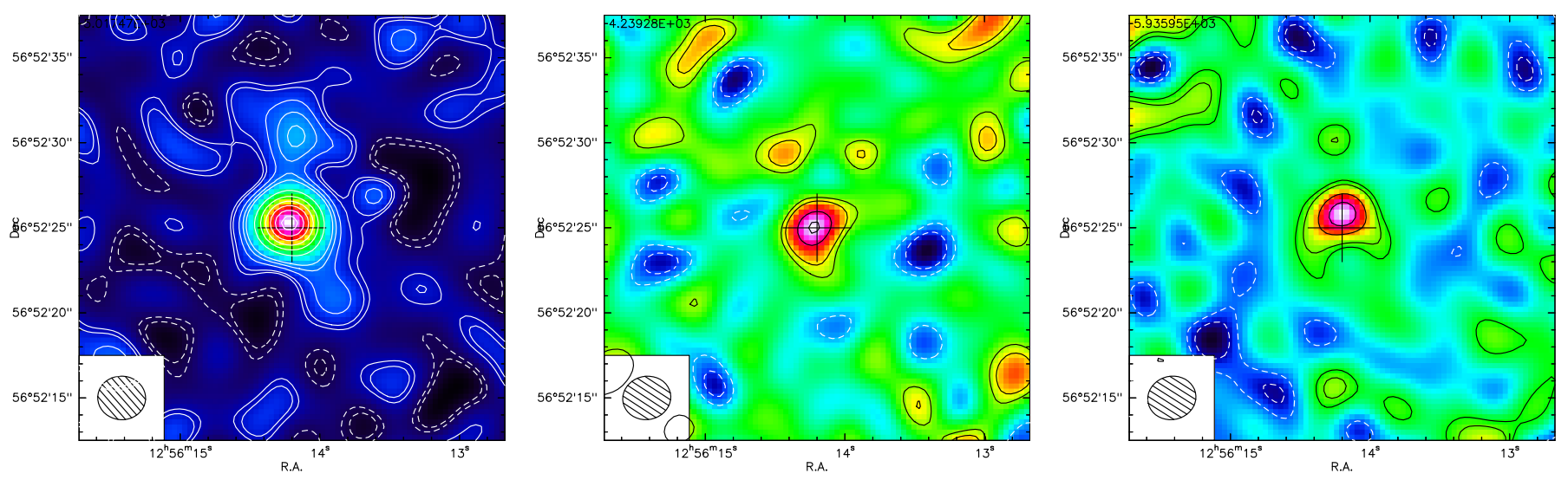

Fig. 1. IRAM PdBI continuum-subtracted maps of the $\mathrm{CN}(1-0)$ line emission. From left to right: (i) total emission integrated between $-800<$ $v\left[\mathrm{~km} \mathrm{~s}^{-1}\right]<1600$, where velocities are calculated with respect to the $J=3 / 2-1 / 2$ spingroup (Table 1); (ii) blue wing of the $J=3 / 2-1 / 2$ line $\left(-500<v\left[\mathrm{~km} \mathrm{~s}^{-1}\right]<-300\right)$; (iii) red wing, integrated between $108.372<v_{\mathrm{obs}}[\mathrm{GHz}]<108.463$, corresponding to velocities $300<v\left[\mathrm{~km} \mathrm{~s}^{-1}\right]<$ 550 with respect to the central frequency of the fainter $J=1 / 2-1 / 2$ spingroup (where the red line wing is not affected by blending). All maps are corrected for the primary beam (45.6" FWHM) and show the central $25^{\prime \prime} \times 25^{\prime \prime}$ region. The synthesised beam is reported in the bottom left corner of the maps. Negative contours (dashed lines) correspond to $(-6 \sigma,-3 \sigma,-2 \sigma)$, and positive contours (solid lines) correspond to ( $2 \sigma, 3 \sigma, 6 \sigma, 9 \sigma$, $n \times 12 \sigma$ ), with $n=1$, 2, etc., where $\sigma$ is the rms of the cleaned datacube (see Table 1).

which the molecular disc is embedded (e.g. Downes \& Solomon 1998), we identify a northern component, detected in $\mathrm{CN}$ at the $12 \sigma$ level, which is spatially extended and offset by $\sim 3-7 \mathrm{kpc}$ with respect to the nucleus of Mrk 231 (left panel of Fig. 1). To our knowledge, this is the first detection of such an extended molecular gas structure outside the main disc of Mrk 231. In Appendix A we show the $\mathrm{CN}(1-0)$ and $\mathrm{CO}(1-0)$ spectra extracted from a region encompassing the northern $\mathrm{CN}$ feature. The properties of this structure, that is, its spectral line profile, spatial extent, and $\mathrm{CN} / \mathrm{CO}(1-0)$ line luminosity ratio, suggest that it may represent an $>5 \mathrm{kpc}$ size extension of the molecular outflow. Further supporting this interpretation, we note that previous $\mathrm{CO}$ and $\mathrm{HCN}$ observations have documented blue- and redshifted features that extend $\sim 2 \mathrm{kpc}$ north of the nucleus of Mrk 231 (Cicone et al. 2012; Aalto et al. 2012; Feruglio et al. $2015)$, that is, in a region lying between the newly discovered northern $\mathrm{CN}$ structure and the well-known central kiloparsec component of the outflow.

The total $\mathrm{CN}(1-0)$ spectrum of Mrk 231 is presented in Fig. 2, together with the $\mathrm{CO}(1-0), \mathrm{HNC}(1-0), \mathrm{HCO}^{+}(1-0), \mathrm{HCN}$ (1-0), $\mathrm{CO}(2-1), \mathrm{HCN}(2-1), \mathrm{CO}(3-2)$, and $\mathrm{HCN}(3-2)$ line spectra. The sizes of the emitting regions differ slightly for the different transitions, which is due to a combination of (i) different beam sizes, where higher resolution data (e.g. taken with configurations $\mathrm{A}$ and $\mathrm{B}$ of the IRAM PdBI, see Table 1) have lower sensitivity to large-scale structures because of interferometric filtering, and (ii) physical conditions, where higher excitation lines tend to be more compact than $\mathrm{CO}(1-0)$, as has been noted for the outflow of Mrk 231 by Cicone et al. (2012). For this reason, we employed slightly different aperture sizes for different tracers, chosen to maximise the total emission detected in the corresponding primary beam-corrected datacubes. More specifically, the spectra shown in Fig. 2 were extracted from circular apertures with diameters of $10^{\prime \prime}$ for $\mathrm{CO}(1-0), \mathrm{CN}(1-0), \mathrm{CO}(2$ $1)$, and $\mathrm{HCN}(2-1) ; 5^{\prime \prime}$ for $\mathrm{CO}(3-2) ; 4^{\prime \prime}$ for $\mathrm{HCN}(1-0), \mathrm{HCO}^{+}$ (1-0), and $\mathrm{HNC}(1-0)$; and $3^{\prime \prime}$ for $\mathrm{HCN}(3-2)$. We note, however, that adopting the same $10^{\prime \prime}$ size aperture for all lines would not change our results, but would just result in slightly noisier $\mathrm{HCN}(1-0), \mathrm{HNC}(1-0), \mathrm{HCO}^{+}(1-0)$, and $\mathrm{HCN}(3-2)$ spectra.

The striking similarity between the spectral profiles of all these different molecular transitions allowed us to perform a simultaneous fit, whose results are overlaid on the data in Fig. 2. The fit employed three Gaussian components that were tied to have the same velocity and width for each line. This simultaneous fitting procedure allowed us to robustly deblend the broad wings of the two $\mathrm{CN}$ spingroups. The amplitude ratio between 

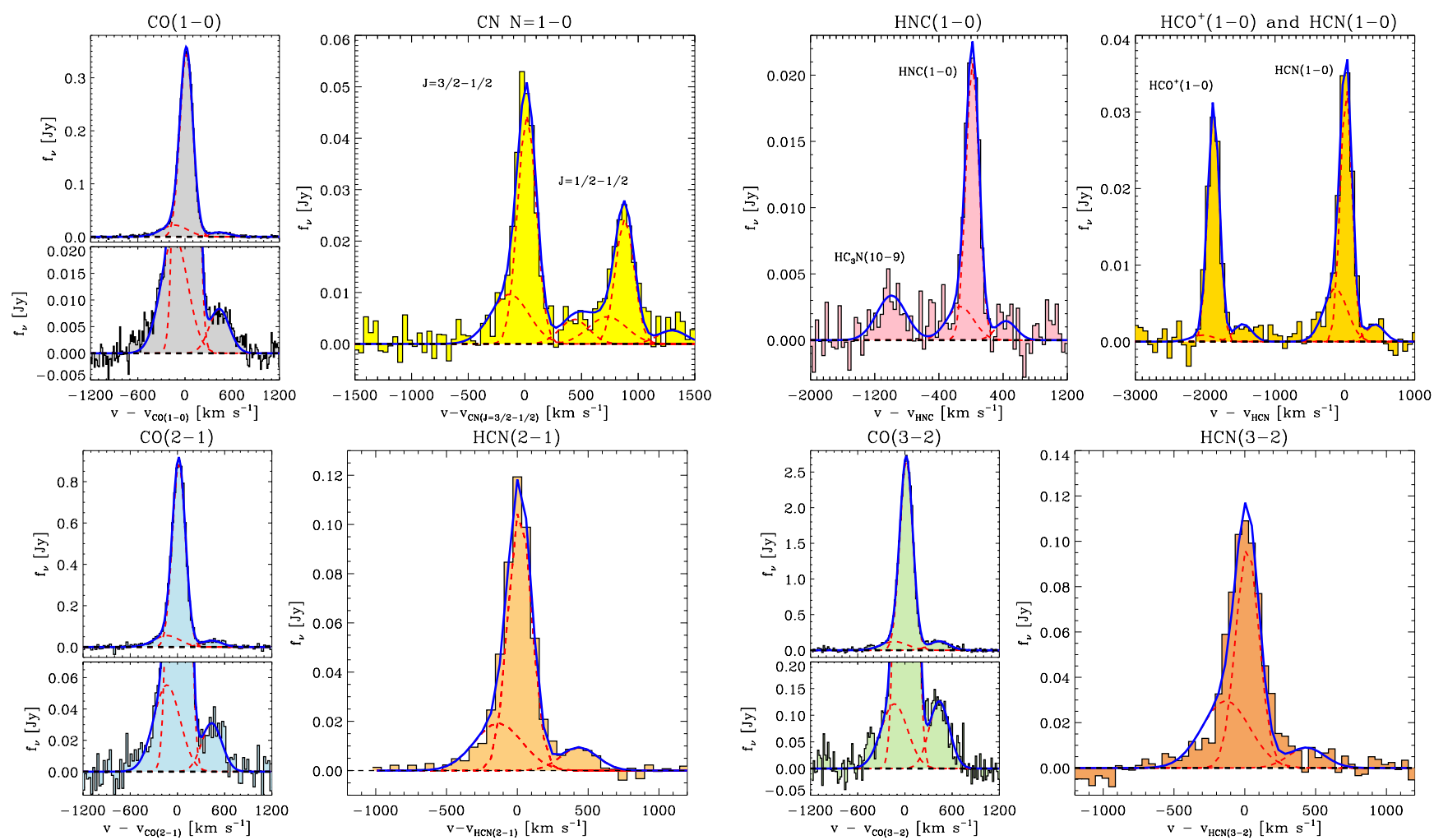

Fig. 2. Total spectra and simultaneous fit of the $\mathrm{CO}(1-0), \mathrm{CN}(1-0), \mathrm{HNC}(1-0), \mathrm{HCO}^{+}(1-0), \mathrm{HCN}(1-0), \mathrm{CO}(2-1), \mathrm{HCN}(2-1)$, $\mathrm{CO}(3-2)$, and HCN(3-2) molecular lines in Mrk 231. Overlaid on the data, we show the individual Gaussians employed in the simultaneous fit (up to three components for each line, red dashed curves) and the total best-fit curve (blue solid lines). Only the fainter $\mathrm{HC}_{3} \mathrm{~N}(10-9)$ line was fitted by a single Gaussian with unconstrained velocity and width. The rms values per spectral channel are $1.7 \mathrm{mJy}$ per $\delta v=14 \mathrm{~km} \mathrm{~s}^{-1}(\mathrm{CO}(1-0))$; $2.3 \mathrm{mJy}$ per $\delta v=43 \mathrm{~km} \mathrm{~s}^{-1}(\mathrm{CN}(1-0)) ; 1.7 \mathrm{mJy} \operatorname{per} \delta v=40 \mathrm{~km} \mathrm{~s}^{-1}(\mathrm{HNC}(1-0)) ; 1.4 \mathrm{mJy}$ per $\delta v=70 \mathrm{~km} \mathrm{~s}^{-1}\left(\mathrm{HCO}^{+}\right.$and $\left.\mathrm{HCN}(1-0)\right) ; 8 \mathrm{mJy}$ per $\delta v=26 \mathrm{~km} \mathrm{~s}{ }^{-1}$ $(\mathrm{CO}(2-1)) ; 1.2 \mathrm{mJy}$ per $\delta v=60 \mathrm{~km} \mathrm{~s}^{-1}(\mathrm{HCN}(2-1)) ; 26 \mathrm{mJy}$ per $\delta v=18 \mathrm{~km} \mathrm{~s}^{-1}(\mathrm{CO}(3-2))$; and $5 \mathrm{mJy}$ per $\delta v=47 \mathrm{~km} \mathrm{~s}^{-1}(\mathrm{HCN}(3-2))$.

Table 2. Line fluxes resulting from the simultaneous fit to the total integrated spectra.

\begin{tabular}{lcccc}
\hline \hline Transition & $\begin{array}{c}\text { Narrow core } \\
{\left[\mathrm{Jy} \mathrm{km} \mathrm{s}^{-1}\right]}\end{array}$ & $\begin{array}{c}\text { Blue wing } \\
{\left[\mathrm{Jy} \mathrm{km} \mathrm{s}^{-1}\right]}\end{array}$ & $\begin{array}{c}\text { Red wing } \\
{\left[\mathrm{Jy} \mathrm{km} \mathrm{s}^{-1}\right]}\end{array}$ & $\begin{array}{c}\text { Total line } \\
{\left[\mathrm{Jy} \mathrm{km} \mathrm{s}^{-1}\right]}\end{array}$ \\
\hline $\mathrm{CO}(1-0)$ & $71.0 \pm 0.6$ & $9.2 \pm 0.7$ & $2.6 \pm 0.2$ & $83.0 \pm 1.0$ \\
$\mathrm{CN}(1-0) J=3 / 2-1 / 2$ & $9.2 \pm 0.4$ & $4.1 \pm 0.5$ & $1.5 \pm 0.3$ & $14.8 \pm 0.8$ \\
$\mathrm{CN}(1-0) J=1 / 2-1 / 2$ & $5.0 \pm 0.3$ & $2.2 \pm 0.3$ & $0.8 \pm 0.2$ & $8.1 \pm 0.5$ \\
$\mathrm{HNC}(1-0)$ & $4.3 \pm 0.3$ & $1.1 \pm 0.4$ & $0.5 \pm 0.2$ & $5.8 \pm 0.5$ \\
$\mathrm{HCO}^{+}(1-0)$ & $6.4 \pm 0.3$ & $0.4 \pm 0.4$ & $0.7 \pm 0.3$ & $7.5 \pm 0.6$ \\
$\mathrm{HCN}(1-0)$ & $6.9 \pm 0.3$ & $2.9 \pm 0.4$ & $0.7 \pm 0.3$ & $10.6 \pm 0.6$ \\
$\mathrm{CO}(2-1)$ & $182.0 \pm 1.7$ & $23 \pm 2$ & $9.9 \pm 1.1$ & $215 \pm 3$ \\
$\mathrm{HCN}(2-1)$ & $22.0 \pm 0.5$ & $8.1 \pm 0.6$ & $3.0 \pm 0.3$ & $33.2 \pm 0.8$ \\
$\mathrm{CO}(3-2)$ & $545 \pm 4$ & $51 \pm 5$ & $40 \pm 4$ & $637 \pm 8$ \\
$\mathrm{HCN}(3-2)$ & $20.1 \pm 1.0$ & $12.5 \pm 1.4$ & $2.9 \pm 0.8$ & $35.4 \pm 1.9$ \\
\hline $\mathrm{HC}{ }_{3} \mathrm{~N}(10-9)$ & \multicolumn{3}{c}{$1.5 \pm 0.5$} \\
\hline
\end{tabular}

Notes: ${ }^{(*)}$ Narrow core best-fit kinematics: $v=18.0 \pm 0.2 \mathrm{~km} \mathrm{~s}^{-1} ; \sigma_{v}=82.3 \pm 0.3 \mathrm{~km} \mathrm{~s}^{-1} .{ }^{(\dagger)}$ Blue wing best-fit kinematics: $v=-135 \pm 16 \mathrm{~km} \mathrm{~s}{ }^{-1}$; $\sigma_{\mathrm{v}}=168 \pm 8 \mathrm{~km} \mathrm{~s}^{-1}$. () Red wing best-fit kinematics: $v=437 \pm 7 \mathrm{~km} \mathrm{~s}^{-1} ; \sigma_{\mathrm{v}}=128 \pm 7 \mathrm{~km} \mathrm{~s}^{-1}$. The $\mathrm{HC}_{3} \mathrm{~N}(10-9)$ line is modelled by a single Gaussian, with unconstrained width and velocity, shifted by $v=-990 \pm 50 \mathrm{~km} \mathrm{~s}^{-1}$ with respect to the HNC(1-0) line, with $\sigma_{\mathrm{v}}=180 \pm 50 \mathrm{~km} \mathrm{~s}^{-1}$ and $S^{\text {peak }}=3.4 \pm 0.8 \mathrm{mJy}$.

the $J=1 / 2-1 / 2$ and $J=3 / 2-1 / 2$ components was fixed for each Gaussian, and constrained by the fit to be $0.55 \pm 0.03$, which is close to the theoretical optically thin value $(0.5)$, as has been noted by Henkel et al. (2014) using single-dish CN(1-0) observations of Mrk 231 with a lower signal-to-noise ratio $(\mathrm{S} / \mathrm{N})$. All other best-fit values are listed in Table 2.

Figure 2 shows that the broad wings are significantly more prominent in the dense gas tracers (e.g. $\mathrm{CN}$ and $\mathrm{HCN}$ ) than in (low-J) $\mathrm{CO}$ transitions. For example, the $\mathrm{CN}(1-0)$ and $\mathrm{HCN}$ (1-0) wings-to-core flux ratios are higher by a factor of four and three, respectively, than the $\mathrm{CO}(1-0)$ ratio.

The flux enhancement of high-density tracers in the outflow of Mrk 231 is further explored in Fig. 3, where we plot separately for the three Gaussian components fitted to the Mrk 231 lines the $\mathrm{CN} / \mathrm{CO}(J=1-0)$ against the $\mathrm{HNC} / \mathrm{CO}, \mathrm{HCO}^{+} / \mathrm{CO}$, and $\mathrm{HCN} / \mathrm{CO}(J=1-0)$ line luminosity ratios (i.e. the ratios 

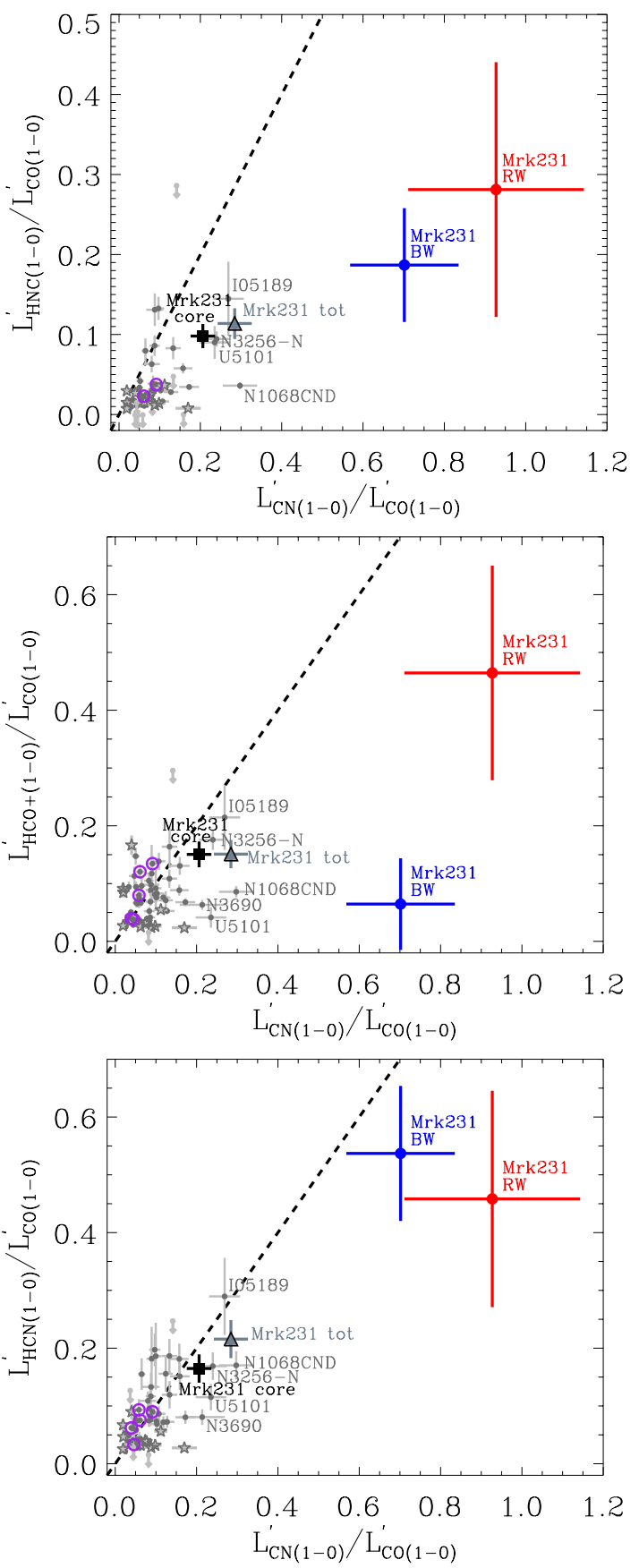

Fig. 3. $\mathrm{CN} / \mathrm{CO}(1-0)$ vs. $\mathrm{HNC} / \mathrm{CO}(1-0)$ (top), $\mathrm{HCO}^{+} / \mathrm{CO}(1-0)$ (middle), and $\mathrm{HCN} / \mathrm{CO}(1-0)$ (bottom) line luminosity ratios for the different line components of Mrk 231 (narrow core, blue wing, red wing, and total emission), including literature samples of nearby galaxies (grey circles) and GMCs (grey stars). Measurements corresponding to extragalactic molecular outflows (NGC 253 and NGC 3256, see Table B.1) are highlighted with purple circles. The $\mathrm{CN}(1-0)$ luminosity corresponds to the sum of the two spingroup components. When only the brightest $J=3 / 2-1 / 2$ spingroup line flux is available in the literature, we extrapolated the total $\mathrm{CN}(1-0)$ flux by multiplying this value by 1.5 . The dashed line indicates the 1:1 relation. All error bars include an 10\% calibration uncertainty, which was added in quadrature to the statistical error $(1 \sigma)$. Upper limits are shown at the $3 \sigma$ level. Sources with $L_{\mathrm{CN}(1-0)}^{\prime} / L_{\mathrm{CO}(1-0)}^{\prime}>0.2$ are labelled in the plot.

between the line luminosities $L_{\text {line }}^{\prime}$ in units of $\left[\mathrm{K} \mathrm{km} \mathrm{s}^{-1} \mathrm{pc}^{2}\right]$, calculated using the definition by Solomon \& Vanden Bout (2005), which are equivalent to brightness temperature ratios). For comparison, Fig. 3 also includes a sample of nearby galaxies as well as Galactic and extragalactic GMCs drawn from the literature (all literature values are reported in Table B.1). Most of the literature data points correspond to integrated line measurements and do not distinguish between disc and outflow components. The only exceptions are galaxies NGC 253 and NGC 3256, for which molecular line fluxes have been measured at different locations by Meier et al. (2015) and Harada et al. (2018), including a few positions coincident with their molecular outflows (highlighted with purple circles in Fig. 3).

It is evident from Fig. 3 that $L_{\mathrm{CN}(1-0)}^{\prime} / L_{\mathrm{CO}(1-0)}^{\prime}$ is significantly boosted in both the blue- and red-shifted sides of the outflow of Mrk 231 compared to the value measured in the narrow core of the lines, which is expected to mainly trace the molecular disc. Higher values than in the core are also measured in $L_{\mathrm{HNC}(1-0)}^{\prime} / L_{\mathrm{CO}(1-0)}^{\prime}$ and $L_{\mathrm{HCN}(1-0)}^{\prime} / L_{\mathrm{CO}(1-0)}^{\prime}$ for both line wings and in $L_{\mathrm{HCO}^{+}(1-0)}^{\prime} / L_{\mathrm{CO}(1-0)}^{\prime}$ for the red wing (but not for the blue wing). However, among all line ratios we explored here, $L_{\mathrm{CN}(1-0)}^{\prime} / L_{\mathrm{CO}(1-0)}^{\prime}$ shows the largest enhancement in the wings relative to the core of the lines.

Furthermore, the $L_{\mathrm{CN}(1-0)}^{\prime} / L_{\mathrm{CO}(1-0)}^{\prime}$ values of $0.70 \pm 0.13$ and $0.9 \pm 0.2$ measured in the blue and red wings, respectively, are significantly higher than the corresponding $L_{\mathrm{HNC}(1-0)}^{\prime} / L_{\mathrm{CO}(1-0)}^{\prime}$ $(0.19 \pm 0.07$ and $0.28 \pm 0.16$ for the blue and red wings), $L_{\mathrm{HCO}+(1-0)}^{\prime} / L_{\mathrm{CO}(1-0)}^{\prime}(0.46 \pm 0.19$ for the red wing $)$ and $L_{\mathrm{HCN}(1-0)}^{\prime} / L_{\mathrm{CO}(1-0)}^{\prime}$ values $(0.54 \pm 0.12$ and $0.46 \pm 0.19$ for the blue and red wings). This means that $\mathrm{CN}$ shows the highest luminosity advantage in the line wings with respect to $\mathrm{CO}$. Quite strikingly, the red wing of Mrk 231 is as bright in $\mathrm{CN}(1-0)$ as in $\mathrm{CO}(1-0)$ emission. High $\mathrm{CN} / \mathrm{CO}$ line luminosity ratios like this are unprecedented when compared to any other extragalactic or Galactic measurement available in the literature. In particular, neither NGC 253 nor NGC 3256 show an enhancement of $\mathrm{CN}$ (or other dense gas tracers) in the outflow that would be comparable to that seen in Mrk 231, suggesting that the physical state of the molecular gas in outflow can differ significantly from source to source. The $\mathrm{CN}(1-0)$ line emission is exceptionally bright in the outflow of Mrk 231, not only compared to the molecular disc of Mrk 231 itself (traced by the core component shown in Fig. 3), but also compared to any other known astrophysical environment.

Figure 3 shows that the emission from high density tracers is clearly boosted with respect to the $\mathrm{CO}(1-0)$ line emission (which should be a good proxy of the total $\mathrm{H}_{2}$ gas mass) in the outflow of Mrk 231. However, the line luminosity ratios computed between different dense gas tracers (e.g. $\mathrm{CN} / \mathrm{HCN}, \mathrm{HNC} / \mathrm{HCN}$ ) are similar as in other known astrophysical sources. Figure 4 shows that the $L_{\mathrm{CN}(1-0)}^{\prime} / L_{\mathrm{HCN}(1-0)}^{\prime}$ and $L_{\mathrm{HNC}(1-0)}^{\prime} / L_{\mathrm{HCN}(1-0)}^{\prime}$ values measured in the wings and in the core of the Mrk 231 lines are formally consistent with each other (although $L_{\mathrm{CN}(1-0)}^{\prime} / L_{\mathrm{HCN}(1-0)}^{\prime}$ is slightly higher in the red wing than in the other components). Furthermore, the two line luminosity ratios fall within the range of values measured in the literature sample.

\subsection{Spectrally resolved clumps in the $\mathrm{HCN}$ and $\mathrm{HCO}^{+}$wings}

The broad wings of the $\mathrm{HCN}(1-0)$ and $\mathrm{HCO}^{+}(1-0)$ lines, first detected by Aalto et al. (2012), show several narrow substructures when examined at high spectral resolution. These features are clearly visible in the spectrum extracted from the central beam (containing $\sim 70 \%$ of the total $\mathrm{HCN}(1-0)$ flux), presented in Fig. 5. To analyse the properties of these spectral clumps, we performed a simultaneous fit to the $\mathrm{HCN}(1-0)$ and $\mathrm{HCO}^{+}$ 


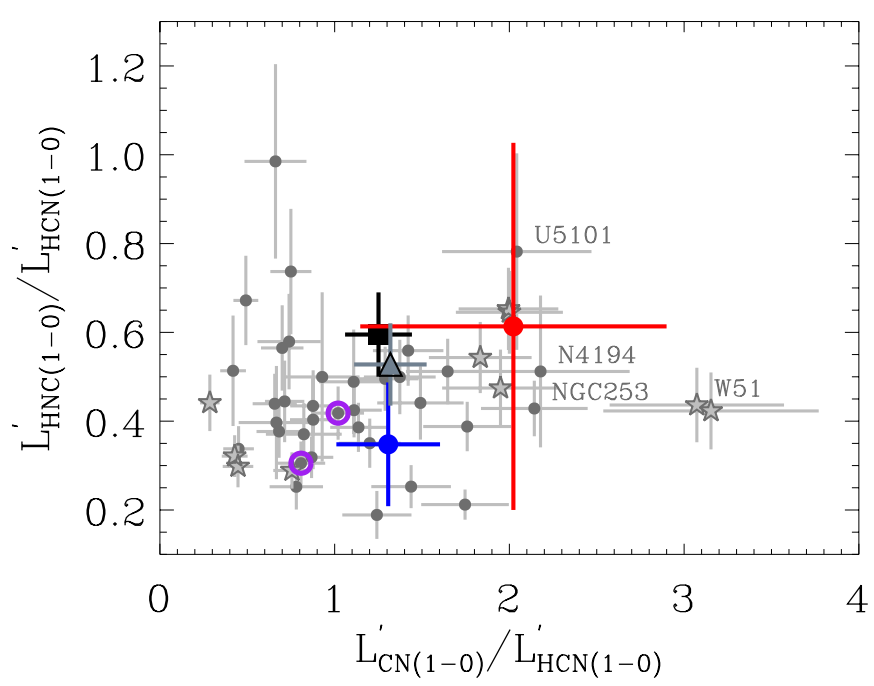

Fig. 4. $\mathrm{CN} / \mathrm{HCN}(1-0)$ vs. $\mathrm{HNC} / \mathrm{HCN}(1-0)$ line luminosity ratios for the different line components of Mrk 231, compared to a sample of local galaxies and Galactic GMCs from the literature. See also caption of Fig. 3.

(1-0) transitions by using multiple Gaussian components, whose results are overlaid on the data in Fig 5. The fit confirms that clumps labelled "A1", "A2", and "A3" are detected in both the $\mathrm{HCN}$ and $\mathrm{HCO}^{+}$lines (with the same velocity and dispersion), but the HCN clumps labelled "B1", "B2", and "B3" do not have a counterpart in $\mathrm{HCO}^{+}$. Only one feature, labelled " $\mathrm{C} 1$ ", is detected in $\mathrm{HCO}^{+}$but not in $\mathrm{HCN}$. As discussed below, all these clumps are detected at high significance. These differences between these species have been pointed out by Lindberg et al. (2016) based on the analysis of the Mrk 231 spectral line profiles. In particular, the authors noted $\mathrm{HCN}$ and $\mathrm{HCO}^{+}$features at different velocities in the line wings. Moreover, their radiative transfer modelling of the $\mathrm{HCN}$ and $\mathrm{HCO}^{+}$line emission is inconsistent when the same outflow properties are used for both species: while a clumpy outflow model could reproduce the HCN emission, the same model would lead to an unrealistically high outflow mass for $\mathrm{HCO}^{+}$. These facts suggest that the $\mathrm{HCN}$ and $\mathrm{HCO}^{+}$line emissions arise in regions with somewhat different physical or chemical properties, which cause the clumpy and chemically differentiated outflow. One possible origin of this difference could be related to the presence of shock fronts in the outflow, with HCN tracing the shocked regions between the outflow and the surrounding gas, and high-velocity $\mathrm{HCO}^{+}$tracing pre-shock gas, closer to the nucleus.

The best-fit Gaussian parameters of the clumps are reported in Table 3. The velocity dispersions of $\sigma_{\mathrm{v}} \sim 7-20 \mathrm{~km} \mathrm{~s}^{-1}$ are consistent with individual GMCs. These $\sigma_{\mathrm{v}}$ values are higher than typical Milky Way GMCs $\left(\left\langle\sigma_{\mathrm{v}}\right\rangle \sim 2 \mathrm{~km} \mathrm{~s}^{-1}\right)$ but lower than those measured for clouds populating the dense nuclear starburst of NGC $253\left(\left\langle\sigma_{\mathrm{v}}\right\rangle \sim 22 \mathrm{~km} \mathrm{~s}^{-1}\right.$, Leroy et al. 2015). By assuming $\mathrm{HCN}-$ to- $\mathrm{H}_{2}$ conversion factors in the range $3.1-24 M_{\odot}\left(\mathrm{K} \mathrm{km} \mathrm{s}^{-1} \mathrm{pc}^{2}\right)^{-1}$ (e.g. Gao \& Solomon 2004; Aalto et al. 2015; Leroy et al. 2015), we compute clumps masses of $M_{\mathrm{mol}} \sim 0.3-4.9 \times 10^{8} M_{\odot}$ (see Table 3$)$. These masses are one order of magnitude higher than typical GMCs in the MW and M 33 (Rosolowsky et al. 2003), but roughly comparable (in the lower-mass end limit) to the more massive GMCs populating the dense environment within the nuclear starburst of NGC 253 (Leroy et al. 2015).

The spectral clumps appear less significant in spectra that are extracted from apertures that are larger than the central beam.
Only features A1, A2, B2, and B3 are also clearly detected in the spectrum extracted from a $4^{\prime \prime}$ aperture, with best-fit $v$ and $\sigma_{\mathrm{v}}$ values consistent with those measured within the central beam (Table 3). The lower significance of the spectral clumps in large apertures arises because they are spatially unresolved at the resolution of the HCN(1-0) data ( 1 kpc, see Table 1$)$. Their flux is therefore maximised in the central beam, but it is washed out in larger apertures because of the superposition with additional outflow components.

We determined the significance of the $\mathrm{HCN}(1-0)$ clumps also in the $u v$ visibility data by analysing the $u v$ amplitude as a function of $u v$ radius ( $u v$ plots). The interferometric images of the $\mathrm{HCN}(1-0)$ clumps and the corresponding $u v$ plots are shown in Fig. 6. The maps and the $u v$ plots, with their flat trend of $u v$ visibilities as a function of $u v$ radius, both confirm that the HCN clumps are spatially unresolved in our observations. A fit to the $u v$ plots using a point source model, whose results are also listed in Table 3 , shows that the HCN clumps (A1-A3, B1-B3) are individually detected at a high significance, ranging from $S / N \sim 4.8-7.4$. Furthermore, as shown in Table 3, the integrated fluxes derived from the $u v$ fit are overall consistent with those obtained from the simultaneous spectral fit shown in Fig. 5. Because the $u v$ visibility data contain the total flux within a certain velocity range (chosen as $v_{\mathrm{c}} \pm 2 \sigma_{\mathrm{v}}$, where $v_{\mathrm{c}}$ and $\sigma_{\mathrm{c}}$ are the central velocity and dispersion of each spectral clump, listed in Table 3) but the spectrum in Fig. 5 only samples the central beam, this flux consistency between the central beam and the $u v$ data suggests that the clumps dominate the HCN flux within the velocity ranges sampled by their narrow line profile. In other words, at the velocity of the clumps, there is little additional flux in more spatially extended and diffuse components.

We also searched for the presence of clumps in the other molecular gas tracers by inspecting the spectra extracted from the central beam. The $\mathrm{HNC}(1-0)$ and $\mathrm{CN}(1-0)$ line wings show clumpy features similar to $\mathrm{HCN}(1-0)$, but the lower $\mathrm{S} / \mathrm{N}$ of these datasets, and in the case of $\mathrm{CN}$, the blending of the two spingroups, do not allow us to reliably fit any of these features. In the $\mathrm{CO}(1-0)$ transition, the line wings are intrinsically much smoother than in the high-density tracers, even when they are studied at the highest available spectral resolution. The only discernible structure in the $\mathrm{CO}(1-0)$ line wings is a redshifted feature with best-fit Gaussian parameters $v=459 \pm 10 \mathrm{~km} \mathrm{~s}^{-1}$, $\sigma_{\mathrm{v}}=39 \pm 17 \mathrm{~km} \mathrm{~s}^{-1}$, and $S_{\mathrm{CO}}^{\text {peak }}=1.7 \pm 0.6 \mathrm{mJy}$, which does not correspond to any of the clumps revealed in $\mathrm{HCN}$ or $\mathrm{HCO}^{+}$.

Our results support a scenario where the molecular outflow entrains high-velocity, dense molecular gas clouds, dominating the emission in the $\mathrm{HCN}$ and $\mathrm{HCO}^{+}$line wings, which are embedded in a more diffuse phase that contributes most to the observed $\mathrm{CO}(1-0)$ line wings. A similar conclusion was independently reached by Cicone et al. (2018) for the dual AGN and LIRG NGC 6240, based on a multi-tracer analysis of the extended molecular outflow.

\section{Discussion}

\subsection{Enhanced $\mathrm{CN} / \mathrm{HCN}$ abundance ratios}

We aim at understanding the origin of the higher $\mathrm{CN} / \mathrm{CO}(1-0)$ and $\mathrm{HCN} / \mathrm{CO}(1-0)$ line luminosity ratios measured in the outflow of Mrk 231 (Fig. 3). This can be caused by a combination of (i) higher fractional abundances of these tracers $\left(X_{\mathrm{HCN}}\right.$, $X_{\mathrm{CN}}$, i.e. the abundance ratios of these species over the total $\mathrm{H}_{2}$ abundance) and/or (ii) a substantial entrainment of dense gas with $n>10^{4} \mathrm{~cm}^{-3}$, which would increase the excitation 


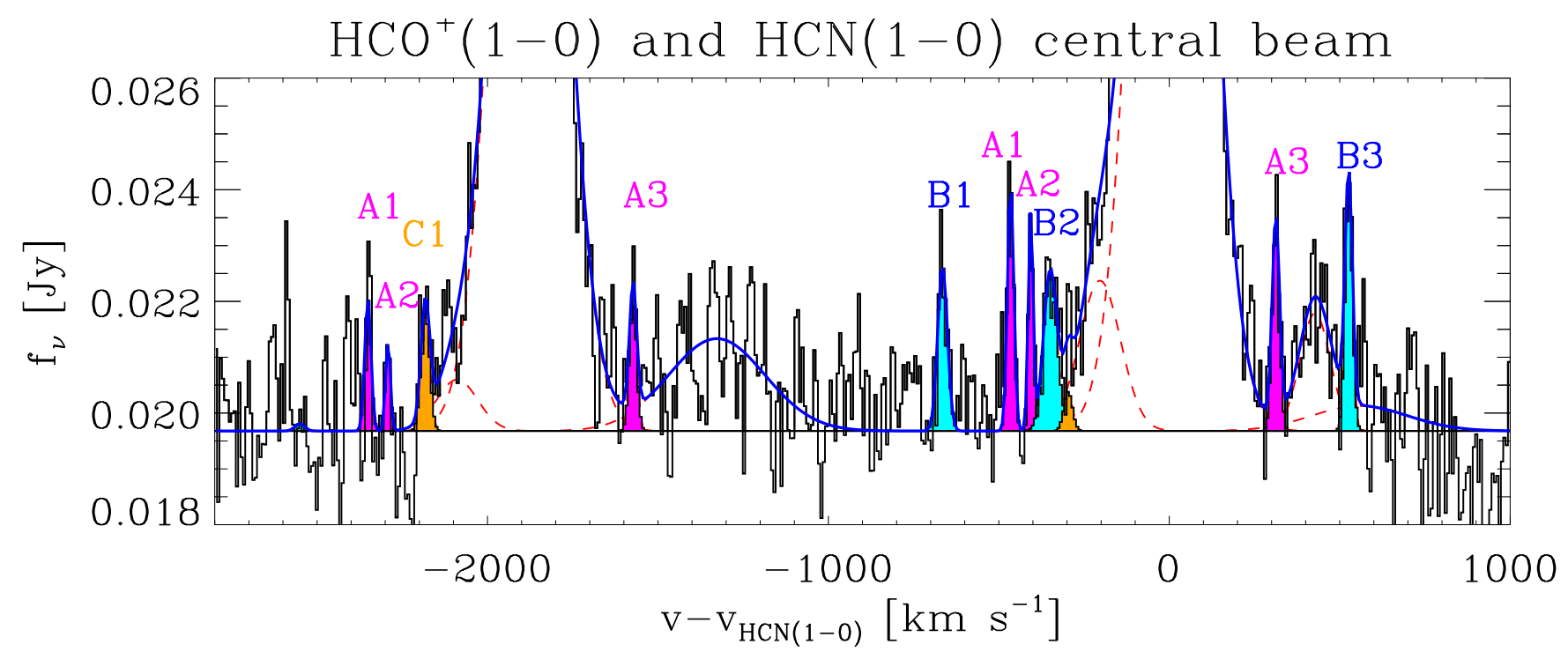

Fig. 5. Spectral fit to the $\mathrm{HCN}(1-0)$ and $\mathrm{HCO}^{+}(1-0)$ line wings showing several spectrally resolved narrow substructures (here often indicated as clumps). The spectrum was extracted from the cleaned datacube before continuum subtraction and using a beam-size aperture centred at $\mathrm{RA}(\mathrm{J} 2000)=12: 56: 14.216$ and $\operatorname{Dec}(\mathrm{J} 2000)=56: 52: 25.186$. The $\mathrm{rms}$ is $1.8 \mathrm{mJy}$ per $\delta v=6.9 \mathrm{~km} \mathrm{~s}^{-1}$ spectral channel. The clump solutions that were simultaneously fitted to both transitions are plotted in magenta, and features detected only in $\mathrm{HCN}\left(\mathrm{HCO}^{+}\right)$are shown in cyan (orange). The best-fit Gaussian parameters are reported in Table 3.

Table 3. Analysis of the $\mathrm{HCN}(1-0)$ and $\mathrm{HCO}^{+}(1-0)$ outflowing clumps.

\begin{tabular}{|c|c|c|c|c|c|c|c|c|c|c|c|}
\hline \multirow[t]{2}{*}{ ID } & \multicolumn{7}{|c|}{ Spectral fit (Gaussian) } & \multicolumn{4}{|c|}{$u v$ visibility fit (point source model) } \\
\hline & $\begin{array}{c}v \\
{\left[\mathrm{~km} \mathrm{~s}^{-1}\right]}\end{array}$ & $\begin{array}{c}\sigma_{\mathrm{v}} \\
{\left[\mathrm{km} \mathrm{s}^{-1}\right]}\end{array}$ & $\begin{array}{c}S_{\text {peak }}^{\mathrm{HCO}+} \\
{[\mathrm{mJy}]}\end{array}$ & $\begin{array}{l}S_{\text {peak }}^{\mathrm{HCN}} \\
{[\mathrm{mJy}]}\end{array}$ & $\begin{array}{c}S / N \\
(\mathrm{HCN})\end{array}$ & $\begin{array}{c}\int S^{\mathrm{HCN}} \mathrm{d} v \\
{\left[\mathrm{Jy} \mathrm{km} \mathrm{s}^{-1}\right]}\end{array}$ & $\begin{array}{c}M_{\mathrm{mol}}{ }^{(\ddagger)} \\
{\left[10^{8} M_{\odot}\right]}\end{array}$ & $\begin{array}{c}\delta \mathrm{RA} \\
{\left[{ }^{\prime \prime}\right]}\end{array}$ & $\begin{array}{c}\delta \text { Dec } \\
{\left[{ }^{\prime \prime}\right]}\end{array}$ & $S / N$ & $\begin{array}{c}\int S^{\mathrm{HCN}} \mathrm{d} v \\
{\left[\mathrm{Jy} \mathrm{km} \mathrm{s}^{-1}\right]}\end{array}$ \\
\hline 1 & $65 \pm 2$ & 9 & $2.4 \pm 0.8$ & 4 & 4.9 & 0.10 & 0 . & $-0.05 \pm 0.13$ & $0.17 \pm 0.12$ & 4.8 & 0.11 \\
\hline $\mathrm{A} 2$ & $-406 \pm 2$ & $7 \pm$ & $1.6 \pm 0.9$ & 3.9 & 3. & 0.07 & & 0.03 & 0.09 & 4.4 & 0.0 \\
\hline A3 & $313 \pm 3$ & $11 \pm 3$ & $2.2 \pm 0.8$ & $3.6 \pm 0.8$ & 4.5 & $0.10 \pm 0.03$ & $0.4-3.4$ & $0.11 \pm 0.10$ & $-0.23 \pm 0.09$ & 6.2 & $0.15 \pm 0.03$ \\
\hline 31 & $-665 \pm 4$ & $14 \pm 4$ & $<2.1^{(\dagger)}$ & $3.0 \pm$ & 4.3 & 0.11 & $0.5-3.7$ & $-0.28 \pm 0.10$ & -0.16 & 6.6 & 0.03 \\
\hline 32 & $-349 \pm 6$ & $20 \pm 6$ & $<2.1^{(\dagger)}$ & $2.8 \pm$ & 4.0 & 0.14 & .9 & $-0.12=$ & 0.12 & 7.1 & 0.2 \\
\hline B3 & $526 \pm 3$ & $11 \pm 3$ & $<2.7^{(\dagger)}$ & $4.1 \pm 0.9$ & 4.6 & $0.11 \pm 0.04$ & $0.5-3.9$ & $-0.11 \pm 0.09$ & $0.10 \pm 0.08$ & 7.4 & $0.16 \pm 0.02$ \\
\hline $\mathrm{C} 1$ & $-297 \pm 6$ & $13 \pm 6$ & $2.1 \pm 0.8$ & $<2.4^{(\dagger)}$ & $<3$ & $<0.07^{(\dagger)}$ & $<2.0^{(\dagger)}$ & & & & \\
\hline
\end{tabular}

Notes. ${ }^{\dagger} 3 \sigma$ upper limit; $\stackrel{(\doteqdot)}{(亠}$ range of dense molecular gas masses estimated from the $\mathrm{HCN}(1-0)$ line luminosity following $M_{\text {mol }}\left[M_{\odot}\right]=$ $\alpha_{\mathrm{HCN}} L_{\mathrm{HCN}(1-0)}^{\prime}\left[\mathrm{K} \mathrm{km} \mathrm{s}^{-1} \mathrm{pc}^{2}\right]$, assuming $\alpha_{\mathrm{HCN}} \in[3.1,24] M_{\odot}\left(\mathrm{K} \mathrm{km} \mathrm{s}^{-1} \mathrm{pc}^{2}\right)^{-1}$, where the lower $\alpha_{\mathrm{HCN}}$ value has been proposed by García-Burillo et al. (2012) for (U)LIRGs, and the higher $\alpha_{\mathrm{HCN}}$ has been measured by Leroy et al. (2015) for individual GMCs in the starburst region of NGC 253. We note that both Gao \& Solomon (2004), based on integrated observations of local galaxies and on the assumption that HCN traces very dense gas, and Shimajiri et al. (2017), based on observations of Galactic GMCs (see also Kauffmann et al. 2017) proposed an intermediate value of $\alpha_{\mathrm{HCN}}=10 M_{\odot}\left(\mathrm{K} \mathrm{km} \mathrm{s}^{-1} \mathrm{pc}^{2}\right)^{-1}$.

of these lines (Aalto et al. 2012, 2015). A high abundance of $\mathrm{HCN}$ may be the signature of shocks (e.g. see Aalto et al. 2012 and references therein), while a high $X_{\mathrm{CN}}$ may trace gas that is exposed to a strong UV radiation field (PDR-like behaviour) from massive stars. García-Burillo et al. (2010) proposed that $\mathrm{CN}$ emission is amplified in the vicinity of an AGN as a result of the X-ray radiation field, although there is still no consensus on this result because many AGN hosts show the opposite behaviour, with $L_{\mathrm{CN}}^{\prime} / L_{\mathrm{CO}}^{\prime}$ values that are lower by a factor of 2-3 than the average ratios measured in pure starbursts (Wilson 2018; Pérez-Beaupuits et al. 2007). The prominent CN line wings of Mrk 231 may indicate that the outflow is bathed in a strong UV radiation field that is either due to star formation in the galaxy disc or to new stars that form within the outflow itself. The latter is a scenario proposed by various recent models and simulations (Zubovas et al. 2013; Zubovas \& King 2014; Ishibashi \& Fabian 2012; Ishibashi et al. 2013; El-Badry et al. 2016; Wang \& Loeb 2018; Decataldo et al. 2019). Star formation in the outflow could have major implications because such stars would have highly radial orbit and could, for instance, contribute to the formation of the spheroidal component of galaxies. Observational confirmations of this new mode of star formation have been presented recently (Maiolino et al. 2017; Gallagher et al. 2019; Rodríguez del Pino et al. 2019). Within the context of our findings, young massive stars that formed inside the outflow would provide a much stronger (internal) UV radiation field with respect to the illumination form stars in the disc or even from the AGN. 



Fig. 6. Interferometric maps and corresponding $u v$ visibility plots of the $\mathrm{HCN}(1-0)$ clumps (top rows: $\mathrm{A} 1$, A2, and $\mathrm{A} 3$; bottom rows: $\mathrm{B} 1$, B2, and $\mathrm{B} 3$, see also labels in the top left corner of the maps). The maps show the continuum-subtracted emission integrated between $v_{\mathrm{c}}-2 \sigma_{\mathrm{v}}<v<v_{\mathrm{c}}+2 \sigma_{\mathrm{v}}$, where $v_{\mathrm{c}}$ and $\sigma_{\mathrm{v}}$ are the best-fit central velocity and velocity dispersion obtained from the spectral line fitting (Table 3 ). Each map is $10^{\prime \prime} \times 10^{\prime \prime}$, and the synthesised beam is $1.45^{\prime \prime} \times 1.21^{\prime \prime}$. Contours correspond to $(-3 \sigma,-2 \sigma, 2 \sigma, 3 \sigma, 4 \sigma, 5 \sigma$, and $6 \sigma)$. Below each map we report the corresponding $u v$ plot, binned in intervals of $30 \mathrm{~m}$. The red solid and dashed lines represent the best-fit value and its associated uncertainty obtained using a point source model (Table 3).

By using the RADEX ${ }^{2}$ dense cloud models developed by Aalto et al. (2015) to reproduce the $\mathrm{HCN}(3-2) /(1-0)$ line luminosity ratios in the outflow of Mrk 231, we can attempt to

\footnotetext{
2 RADEX is a publicly available non-local thermodynamic equilibrium radiative transfer code that uses as input parameters the molecular gas column density $\left(N_{\mathrm{H}_{2}}\right)$, the line width $(\Delta v)$, the gas temperature $\left(T_{\text {kin }}\right)$, the background temperature (set equal to the CMB temperature at $z=0$, i.e. $T_{\text {bg }}=2.73 \mathrm{~K}$ ), and the gas volume density (van der Tak et al. 2007).
}

find a combination of $X_{\mathrm{HCN}}, X_{\mathrm{CN}}, T_{\text {kin }}$, and $n_{\mathrm{H}_{2}}$ solutions that can also fit the $\mathrm{CN} / \mathrm{HCN}$ and $\mathrm{CN}$ spin doublet line ratios (Table 2). We assume that the $\mathrm{HCN}$ and $\mathrm{CN}$ line emissions arise from the same dense cloud population, while the low-J CO line emission is due to a different, more diffuse phase of the outflow. We recall that in these models (see also Aalto et al. 2015), the dense clouds can be either self-gravitating virialised clouds, which implies that their internal velocity dispersion $\left(\Delta v_{\mathrm{sg}}\right)$ is locked 
to their mass $\left(M_{\mathrm{vir}}\right)$ and size $(R)$ through $\Delta v_{\mathrm{sg}}=\left(G M_{\mathrm{vir}} / G\right)^{1 / 2}$ or unbound clouds, for which $\Delta v \gg \Delta v_{\mathrm{sg}}$. We explored $\mathrm{CN}$ and $\mathrm{HCN}$ abundances in the range between $10^{-8}$ and $10^{-6}$. We find that depending on whether the clouds are self-gravitating or unbound, the models produce very different values for the absolute $\mathrm{CN}$ and $\mathrm{HCN}$ abundances, hence $X_{\mathrm{CN}}$ and $X_{\mathrm{HCN}}$ remain quantitatively unconstrained for the outflow with current data. However, all possible solutions that fit the observed line ratios consistently require $X_{\mathrm{CN}}>X_{\mathrm{HCN}}$, with a $\mathrm{CN}$ abundance that is at least a factor of three higher than the HCN abundance. Gas densities for this outflow phase (traced by the $\mathrm{CN}$ and $\mathrm{HCN}$ emissions) are $n_{\mathrm{H}_{2}} \sim 10^{5}-10^{6} \mathrm{~cm}^{-3}$, with temperatures not much higher than $T_{\text {kin }} \sim 20 \mathrm{~K}$. Because $\mathrm{CN}$ is a well-known PDR tracer (see also Sect. 1), these results strongly suggest that the whole dense cloud population in outflow is affected by UV radiation. We should mention that high $\mathrm{CN}$ abundances may also be due to cosmic rays (e.g. see work done on the Galactic centre by Harada et al. 2015), which are known to permeate the outflow of Mrk 231, as inferred by González-Alfonso et al (2018) based on the high $\mathrm{OH}^{+}$abundance. However, it is not clear whether a cosmic-ray chemistry would also explain $X_{\mathrm{CN}}>X_{\mathrm{HCN}}$.

In the literature (see Appendix B), there are only two other extragalactic molecular outflows with available $\mathrm{CN}(1-0)$ observations (NGC 3256 and NGC 253, see also Table B.1), and neither of them shows the exceptionally high $L_{\mathrm{CN}(1-0)}^{\prime} / L_{\mathrm{CO}(1-0)}^{\prime}$ values that we measure in Mrk 231. However, in NGC 3256, Sakamoto et al. (2014) and Harada et al. (2018) found an enhancement of $\mathrm{CN}$ and $\mathrm{HCN}$ at the outflow position. Different from $\mathrm{CN}$, there are quite a few extragalactic outflows with $\mathrm{HCN}$ and/or $\mathrm{HCO}^{+}$observations. In Arp 220, observations by Barcos-Muñoz et al. (2018) imply $L_{\mathrm{HCN}(1-0)}^{\prime} / L_{\mathrm{CO}(1-0)}^{\prime} \sim 0.9$ and $\sim 7$ for the blue- and redshifted outflows, which are significantly higher than the corresponding luminosity ratio of $0.20 \pm 0.03$ obtained using the $\mathrm{HCN}$ and $\mathrm{CO}$ emissions integrated across the whole galaxy (Table B.1). In IC 5063, the high $\mathrm{HCO}^{+}(4-3) / \mathrm{CO}(2-1)$ ratio measured in the outflow by Oosterloo et al. (2017) points to an additional dense component with $n \gtrsim 10^{6} \mathrm{~cm}^{-3}$ that is not accounted for by the low- $J$ $\mathrm{CO}$ emission (which is likely optically thin, e.g. Dasyra et al. 2016). Aladro et al. (2018) detected the blueshifted component in the molecular outflow of Mrk 273 in $\operatorname{HCN}(3-2)$ emission, and its location is consistent with the $\mathrm{CO}(1-0)$ component imaged by Cicone et al. (2014). Among other extragalactic outflows detected in dense molecular gas tracers, we list $\mathrm{NGC} 1068\left(\mathrm{HCN}(4-3)\right.$ and $\mathrm{HCO}^{+}(4-3)$ emission, García-Burillo et al. 2014), NGC 1266 (CS(2-1) and HCN(1-0) emission, Alatalo et al. 2015), and IC 860 (CS(7-6) absorption, Aalto et al. 2019).

Interestingly, even though high critical density molecules have been detected in the molecular streamers embedded in the starburst-driven outflows of NGC 253 and M 82, these dense gas tracers do not appear to be enhanced in these outflows. In NGC 253, the CN emission is boosted (with respect to both $\mathrm{CO}$ and $\mathrm{C}^{17} \mathrm{O}$ ) in the central starburst, but not in the streamers (Meier et al. 2015). The HCN/CO luminosity ratio is instead similar between starburst and outflow (Walter et al. 2017). The outflow of M82 was detected in $\mathrm{HCO}^{+}(1-0)$ and HCN(1-0) emission (Salas et al. 2014), but the mass traced by these molecules is a small fraction $(\gtrsim 2 \%)$ of the total (CO-based) mass of the outflow. Hence, the molecular gas filaments embedded in the starburst-driven outflows of NGC 253 and M 82 appear to differ in their physical properties from the outflow of Mrk 231.
The exceptional boost of $\mathrm{CN}$ and $\mathrm{HCN}$ in the outflow of Mrk 231 may hold clues to explain the particularly strong emission from these dense gas tracers in integrated observations of this ULIRG. Although the total molecular line flux of Mrk 231 is dominated by the narrow core rather than by the wings (as also reflected by the line ratios in Fig. 3, where the integrated data point lies closer to the core than to the wings), we note that the $\mathrm{CN} / \mathrm{CO}$ and $\mathrm{HCN} / \mathrm{CO}$ luminosity ratios measured in the narrow core are still quite high compared to the nearby galaxy population and to local GMCs, albeit not as extreme as those measured in the wings. We cannot rule out that the low projected velocity component of the outflow also significantly contaminates the narrow emission (such contribution can be quantified only through very high $\mathrm{S} / \mathrm{N}$ and spatially resolved observations). Because the low projected velocity component of the outflow would share the same enhancement of $\mathrm{CN}$ and $\mathrm{HCN}$ emission as the high-velocity component that is responsible for the wings, it would in this case also boost the line ratios that are measured for the narrow core. In the hypothesis that Mrk 231-like outflows have a chemical effect on the global ISM of their host galaxies, they would resemble scaled-up versions of the so-called chemically active protostellar outflows revealed in young stellar objects, which are characterised by higher abundances of shocksensitive tracers such as $\mathrm{SiO}$ (Tafalla \& Hacar 2013) or even CN and $\mathrm{HCO}^{+}$(Bachiller et al. 2001).

\subsection{Dense clumps embedded in the outflow}

The discovery of narrow features in the $\mathrm{HCN}$ and $\mathrm{HCO}^{+}$line wings suggests that the outflow embeds coherent dense molecular gas complexes, with velocity dispersions similar to extragalactic GMCs detected in dense environments. Furthermore, our data show that while these clumps dominate the highvelocity emission from the high-density tracers, they are absent from the wings of low- $J$ CO lines. The HCN clouds embedded in the outflow of Mrk 231 are massive, with inferred $M_{\text {mol }}$ values in the range $\sim 0.3-4.9 \times 10^{8} M_{\odot}$ (Table 3 ), and have large velocity dispersions of $\sim 7-20 \mathrm{~km} \mathrm{~s}^{-1}$. These unusually high masses may result from an observational bias, where multiple outflowing clouds with overlapping line-of-sight velocities contribute to the narrow sub-structures that we identify in the (beam-averaged) HCN spectra in Fig. 5. However, it is also possible that we witness the survival of only the largest and/or the most massive molecular clouds in outflow, which are less affected by photoevaporation (Decataldo et al. 2019) and hydrodynamical instabilities (Gronke \& Oh 2018; Armillotta et al. 2017).

These physical properties, that is, masses and velocity dispersions higher by a factor of ten than GMCs in the Galactic disc, seem to be more typical of molecular clouds that form in dense environments, such as those residing in the nuclear starburst of NGC 253. The latter, identified by Leroy et al. (2015) through HCN-to-CO intensity peaks (one of which may even be entrained in the outflow), are characterised by higher surface and volume densities, shorter free-fall timescales and accordingly, higher star formation efficiencies than typical disc GMCs. That the HCN clumps detected in the outflow of Mrk 231 correspond to star-forming complexes is a very interesting prospect, especially in the context of the new scenarios predicting the formation of stars inside outflows. Dense and clumpy gas is a condition that typically results in rapid gravitational collapse and star formation (Decataldo et al. 2019); our finding therefore provides further support for this new mode of star formation.

We note that if the cloud mass distribution in outflow differs strongly from that of typical GMCs, as suggested by our 
results, it would result in a different initial mass function (IMF) of the stars that would form within such environment (Hopkins 2012), and might explain other exotic properties of (U)LIRGs such as their high ${ }^{18} \mathrm{O}$ abundance (which favours a top-heavy IMF, e.g. Brown \& Wilson 2019). However, a valid objection is that even if the high-velocity clumps trace individual clouds, we know that at least in the Milky Way, not all GMCs are active stellar nurseries. Even within GMCs, HCN emission can be quite widespread, and up to $\sim 50 \%$ of it can be associated with extended regions with $n_{\mathrm{H}_{2}} \lesssim 10^{3} \mathrm{~cm}^{-3}$, which is two orders of magnitude below the critical density for collisions with $\mathrm{H}_{2}$ molecules (Pety et al. 2017; Kauffmann et al. 2017; Harada et al. 2019). In these regions, HCN may be excited by electron collisions (Goldsmith \& Kauffmann 2017). Additional data are required to settle this issue.

\section{Summary and conclusions}

We have reported the detection of an exceptional enhancement of $\mathrm{CN}(1-0)$ line emission in the molecular outflow of Mrk 231. More specifically, we measured $L_{\mathrm{CN}(1-0)}^{\prime} / L_{\mathrm{CO}(1-0)}^{\prime}$ values of $0.70 \pm 0.13$ (blueshifted wing) and $0.9 \pm 0.2$ (redshifted wing), which are not only far higher than the value measured in the narrow core component $(0.21 \pm 0.03)$, but also higher than any other known Galactic or extragalactic source. The $\mathrm{CN} / \mathrm{CO}(1-0)$ line luminosity ratios computed for the line wings are also significantly higher than the respective $\mathrm{HCN} / \mathrm{CO}(1-0)$, $\mathrm{HCO}^{+} / \mathrm{CO}(1-0)$ and $\mathrm{HNC} / \mathrm{CO}(1-0)$ line luminosity ratios, indicating that among the dense gas tracers that have so far been explored in the outflow of Mrk 231, CN has the highest luminosity advantage with respect to $\mathrm{CO}$.

We have found that in order to reproduce these ratios, the dense gas cloud population entrained in the outflow must be characterised by an $\mathrm{CN}$ abundance that is at least a factor of three higher than the HCN abundance, by gas densities of $n_{\mathrm{H}_{2}} \sim 10^{5-6} \mathrm{~cm}^{-3}$, and kinetic temperatures not much higher than $T_{\text {kin }} \sim 20 \mathrm{~K}$. This result can be interpreted (although not uniquely) in terms of an outflow chemistry that is heavily affected by UV radiation because $\mathrm{CN}$ emission has been found to be amplified mainly in PDRs. A population of young massive stars formed inside the outflow, as predicted by recent models, would naturally provide an explanation for this strong UV radiation field.

Only two other extragalactic molecular outflows with available $\mathrm{CN}$ measurements have been reported before, NGC 253 and NGC 3256, and neither of them shows an enhancement of CN similar to Mrk 231. Although we are still limited by statistics, we may conclude that the outflow of Mrk 231 is rather exceptional in its chemical and physical properties. In the future, using deeper observations and higher spatial resolution, it would be interesting to explore whether the outflow component is responsible for the exceptionally strong emission from dense $\mathrm{H}_{2}$ gas tracers observed in the global ISM of this ULIRG.

Additionally, we have shown that the $\operatorname{HCN}(1-0)$ and $\mathrm{HCO}^{+}(1-0)$ line wings are spectrally resolved into several narrow sub-structures with velocity dispersions of $\sigma_{\mathrm{v}} \sim 7-$ $20 \mathrm{~km} \mathrm{~s}^{-1}$ and inferred masses of $M_{\mathrm{mol}} \sim 0.3-4.9 \times 10^{8} M_{\odot}$, which we interpret as individual dense clumps entrained in the outflow of Mrk 231. These properties are consistent with the most massive extragalactic GMCs populating the nuclear starburst of NGC 253. Three of the outflowing clumps are detected simultaneously in both the $\mathrm{HCN}$ and $\mathrm{HCO}^{+}(1-0)$ transitions, while an additional three (one) are detected only in $\mathrm{HCN}$ (or $\mathrm{HCO}^{+}$), supporting the hypothesis of a chemically differentiated outflow. An analysis of the $u v$ visibility data indicates that these narrow HCN outflow features are spatially unresolved at the resolution of our data $(\sim 1 \mathrm{kpc})$, but at their line-of-sight velocity, there is little additional HCN flux in more spatially extended and diffuse components. The $\mathrm{CO}$ spectral line wings are instead intrinsically much smoother, implying that low- $J$ CO emission is dominated by a more diffuse component of the outflow.

This discovery strongly suggests that the outflow of Mrk 231 embeds coherent molecular gas cloud complexes, similar to GMCs, but they are more massive and have different chemical properties than the GMCs in the Milky Way. This possibly indicates that we witness the survival of only the largest molecular clouds that are less affected by photoevaporation and hydrodynamical instabilities, which are the processes that we expect to be at work in energetic multiphase outflows and that may quickly destroy most of the coldest and densest gas. If these clumps correspond to star-forming complexes, as predicted by recent theoretical models, the stars that would form from gravitational collapse of these massive clouds would probably follow a different IMF. However, this intriguing hypothesis requires follow-up observations to confirm the star-forming nature of these clumps and to better constrain their masses.

Finally, our interferometric maps have revealed a new molecular gas structure in Mrk 231, offset to the north by $5^{\prime \prime}$. Its high $\mathrm{CN} / \mathrm{CO}$ line luminosity ratio and broad blueshifted line profiles suggest that this northern component may trace an additional extension of the outflow that reaches out to $r>5 \mathrm{kpc}$ from the nucleus.

Acknowledgements. The interpretation of our data, especially for what concerns the ISM chemistry in the presence of strong UV fields, benefited greatly from extensive discussions with the late Malcolm Walmsley. We are deeply grateful to him, not only for his help with this paper, but also for the several insightful and enlightening discussions that some of us had with him on a broad range of topics in Astrophysics. CC thanks Jens Kauffmann for providing the Orion data, Nanase Harada and Kazushi Sakamoto for providing non-published measurements of NGC 3256, Paola Severgnini for insightful discussions about the multi-wavelength emission of Mrk 231, and Paola Caselli for valuable explanations on the properties of $\mathrm{CN}$. We thank the anonymous referee for helpful comments. This work is based on observations carried out under project numbers UA26, TQ2F, UB26, V087, U-D, W028, VQ26, WA85 with the IRAM NOEMA Interferometer. IRAM is supported by INSU/CNRS (France), MPG (Germany) and IGN (Spain). This project has received funding from the European Union's Horizon 2020 research and innovation programme under the Marie Skłodowska-Curie grant agreement No 664931. RM acknowledges ERC Advanced Grant 695671 "QUENCH" and support by the Science and Technology Facilities Council (STFC).

\section{References}

Aalto, S., Polatidis, A. G., Hüttemeister, S., \& Curran, S. J. 2002, A\&A, 381, 783

Aalto, S., Garcia-Burillo, S., Muller, S., et al. 2012, A\&A, 537, A44

Aalto, S., Garcia-Burillo, S., Muller, S., et al. 2015, A\&A, 574, A85

Aalto, S., Muller, S., König, S., et al. 2019, A\&A, 627, A147

Aladro, R., Martín, S., Riquelme, D., et al. 2015, A\&A, 579, A101

Aladro, R., König, S., Aalto, S., et al. 2018, A\&A, 617, A20

Alatalo, K. 2015, ApJ, 801, L17

Alatalo, K., Lacy, M., Lanz, L., et al. 2015, ApJ, 798, 31

Armillotta, L., Fraternali, F., Werk, J. K., Prochaska, J. X., \& Marinacci, F. 2017, MNRAS, 470, 114

Baan, W. A., Henkel, C., Loenen, A. F., Baudry, A., \& Wiklind, T. 2008, A\&A, 477, 747

Bachiller, R., Pérez Gutiérrez, M., Kumar, M. S. N., \& Tafalla, M. 2001, A\&A, 372, 899

Barcos-Muñoz, L., Aalto, S., Thompson, T. A., et al. 2018, ApJ, 853, L28

Biernacki, P., \& Teyssier, R. 2018, MNRAS, 475, 5688

Boger, G. I., \& Sternberg, A. 2005, ApJ, 632, 302

Braito, V., Della Ceca, R., Piconcelli, E., et al. 2004, A\&A, 420, 79

Brown, T., \& Wilson, C. D. 2019, ApJ, 879, 17 
Chevalier, R. A., \& Clegg, A. W. 1985, Nature, 317, 44

Cicone, C., Feruglio, C., Maiolino, R., et al. 2012, A\&A, 543, A99

Cicone, C., Maiolino, R., Sturm, E., et al. 2014, A\&A, 562, A21

Cicone, C., Severgnini, P., Papadopoulos, P. P., et al. 2018, ApJ, 863, 143

Costa, T., Sijacki, D., \& Haehnelt, M. G. 2015, MNRAS, 448, L30

Costa, T., Rosdahl, J., Sijacki, D., \& Haehnelt, M. G. 2018, MNRAS, 479, 2079

Costagliola, F., Aalto, S., Rodriguez, M. I., et al. 2011, A\&A, 528, A30

Dasyra, K. M., Combes, F., Oosterloo, T., et al. 2016, A\&A, 595, L7

Davies, R. I., Müller Sánchez, F., Genzel, R., et al. 2007, ApJ, 671, 1388

Decataldo, D., Pallottini, A., Ferrara, A., Vallini, L., \& Gallerani, S. 2019, MNRAS, 487, 3377

Di Matteo, T., Springel, V., \& Hernquist, L. 2005, Nature, 433, 604

Downes, D., \& Solomon, P. M. 1998, ApJ, 507, 615

El-Badry, K., Wetzel, A., Geha, M., et al. 2016, ApJ, 820, 131

Faucher-Giguère, C.-A., \& Quataert, E. 2012, MNRAS, 425, 605

Feruglio, C., Maiolino, R., Piconcelli, E., et al. 2010, A\&A, 518, L155

Feruglio, C., Fiore, F., Carniani, S., et al. 2015, A\&A, 583, A99

Fischer, J., Sturm, E., González-Alfonso, E., et al. 2010, A\&A, 518, L41

Fluetsch, A., Maiolino, R., Carniani, S., et al. 2019, MNRAS, 483, 4586

Fuente, A., Martin-Pintado, J., Cernicharo, J., \& Bachiller, R. 1993, A\&A, 276, 473

Gallagher, R., Maiolino, R., Belfiore, F., et al. 2019, MNRAS, 485, 3409

Gao, Y., \& Solomon, P. M. 2004, ApJS, 152, 63

García-Burillo, S., Usero, A., Fuente, A., et al. 2010, A\&A, 519, A2

García-Burillo, S., Usero, A., Alonso-Herrero, A., et al. 2012, A\&A, 539, A8

García-Burillo, S., Combes, F., \& Usero, A. 2014, A\&A, 567, A125

Goldsmith, P. F., \& Kauffmann, J. 2017, ApJ, 841, 25

González-Alfonso, E., Fischer, J., Graciá-Carpio, J., et al. 2014, A\&A, 561, A27

González-Alfonso, E., Fischer, J., Bruderer, S., et al. 2018, ApJ, 857, 66

Gronke, M., \& Oh, S. P. 2018, MNRAS, 480, L111

Harada, N., Riquelme, D., Viti, S., et al. 2015, A\&A, 584, A102

Harada, N., Sakamoto, K., Martín, S., et al. 2018, ApJ, 855, 49

Harada, N., Nishimura, Y., Watanabe, Y., et al. 2019, ApJ, 871, 238

Henkel, C., Asiri, H., Ao, Y., et al. 2014, A\&A, 565, A3

Hopkins, P. F. 2012, MNRAS, 423, 2037

Imanishi, M., Nakanishi, K., \& Izumi, T. 2017, ApJ, 849, 29

Ishibashi, W., \& Fabian, A. C. 2012, MNRAS, 427, 2998

Ishibashi, W., Fabian, A. C., \& Canning, R. E. A. 2013, MNRAS, 431, 2350

Ishibashi, W., Fabian, A. C., \& Maiolino, R. 2018, MNRAS, 476, 512

Kauffmann, J., Goldsmith, P. F., Melnick, G., et al. 2017, A\&A, 605, L5

Leighly, K. M., Terndrup, D. M., Baron, E., et al. 2014, ApJ, 788, 123

Leroy, A. K., Bolatto, A. D., Ostriker, E. C., et al. 2015, ApJ, 801, 25

Leroy, A. K., Usero, A., Schruba, A., et al. 2017, ApJ, 835, 217

Lindberg, J. E., Aalto, S., Muller, S., et al. 2016, A\&A, 587, A15

Lípari, S., Terlevich, R., Zheng, W., et al. 2005, MNRAS, 360, 416

Lípari, S., Sanchez, S. F., Bergmann, M., et al. 2009, MNRAS, 392, 1295

Lutz, D., Sturm, E., Janssen, A., et al. 2020, A\&A, 633, A134

Maiolino, R., Russell, H. R., Fabian, A. C., et al. 2017, Nature, 544, 202

Meier, D. S., Walter, F., Bolatto, A. D., et al. 2015, ApJ, 801, 63

Meijerink, R., Spaans, M., \& Israel, F. P. 2007, A\&A, 461, 793

Morganti, R., Veilleux, S., Oosterloo, T., Teng, S. H., \& Rupke, D. 2016, A\&A, 593, A30

Murray, N., Quataert, E., \& Thompson, T. A. 2005, ApJ, 618, 569
Nardini, E., Risaliti, G., Watabe, Y., Salvati, M., \& Sani, E. 2010, MNRAS, 405, 2505

Nims, J., Quataert, E., \& Faucher-Giguère, C.-A. 2015, MNRAS, 447, 3612

Nishimura, Y., Shimonishi, T., Watanabe, Y., et al. 2016, ApJ, 818, 161

Oosterloo, T., Raymond Oonk, J. B., Morganti, R., et al. 2017, A\&A, 608, A38

Papadopoulos, P. P., van der Werf, P. P., Xilouris, E. M., et al. 2012, MNRAS, 426, 2601

Pereira-Santaella, M., Colina, L., García-Burillo, S., et al. 2018, A\&A, 616, A171

Pérez-Beaupuits, J. P., Aalto, S., \& Gerebro, H. 2007, A\&A, 476, 177

Pety, J., Guzmán, V. V., Orkisz, J. H., et al. 2017, A\&A, 599, A98

Piconcelli, E., Miniutti, G., Ranalli, P., et al. 2013, MNRAS, 428, 1185

Planck Collaboration XIII. 2016, A\&A, 594, A13

Privon, G. C., Herrero-Illana, R., Evans, A. S., et al. 2015, ApJ, 814, 39

Richings, A. J., \& Faucher-Giguère, C.-A. 2018, MNRAS, 474, 3673

Rodríguez del Pino, B., Arribas, S., Piqueras López, J., Villar-Martín, M., \& Colina, L. 2019, MNRAS, 486, 344

Rodriguez-Franco, A., Martin-Pintado, J., \& Fuente, A. 1998, A\&A, 329, 1097

Rosolowsky, E., Engargiola, G., Plambeck, R., \& Blitz, L. 2003, ApJ, 599, 258

Rupke, D. S. N., \& Veilleux, S. 2011, ApJ, 729, L27

Sakamoto, K., Aalto, S., Combes, F., Evans, A., \& Peck, A. 2014, ApJ, 797, 90

Salas, P., Galaz, G., Salter, D., et al. 2014, ApJ, 797, 134

Schneider, E. E., Robertson, B. E., \& Thompson, T. A. 2018, ApJ, 862, 56

Scoville, N. Z., \& Solomon, P. M. 1974, ApJ, 187, L67

Shimajiri, Y., André, P., Braine, J., et al. 2017, A\&A, 604, A74

Shirley, Y. L. 2015, PASP, 127, 299

Sijacki, D., Springel, V., Di Matteo, T., \& Hernquist, L. 2007, MNRAS, 380, 877

Silk, J., \& Rees, M. J. 1998, A\&A, 331, L1

Skatrud, D. D., De Lucia, F. C., Blake, G. A., \& Sastry, K. V. L. N. 1983, J. Mol. Spectrosc., 99, 35

Solomon, P. M., \& Vanden Bout, P. A. 2005, ARA\&A, 43, 677

Sturm, E., González-Alfonso, E., Veilleux, S., et al. 2011, ApJ, 733, L16

Tafalla, M., \& Hacar, A. 2013, A\&A, 552, L9

Takano, S., Nakajima, T., \& Kohno, K. 2019, PASJ, 65, 100

Taylor, G. B., Silver, C. S., Ulvestad, J. S., \& Carilli, C. L. 1999, ApJ, 519, 185

Teng, S. H., Veilleux, S., \& Baker, A. J. 2013, ApJ, 765, 95

Teng, S. H., Brandt, W. N., Harrison, F. A., et al. 2014, ApJ, 785, 19

Thompson, T. A., Quataert, E., Zhang, D., \& Weinberg, D. H. 2016, MNRAS, 455,1830

van der Tak, F. F. S., Black, J. H., Schöier, F. L., Jansen, D. J., \& van Dishoeck, E. F. 2007, A\&A, 468, 627

Veilleux, S., Rupke, D. S. N., Kim, D. C., et al. 2009, ApJS, 182, 628

Veilleux, S., Meléndez, M., Sturm, E., Gracia-Carpio, J., Fischer, J., et al. 2013, ApJ, 776, 27

Walter, F., Bolatto, A. D., Leroy, A. K., et al. 2017, ApJ, 835, 265

Wang, X., \& Loeb, A. 2018, New Astron., 61, 95

Watanabe, Y., Sakai, N., Sorai, K., \& Yamamoto, S. 2014, ApJ, 788, 4

Watanabe, Y., Nishimura, Y., Harada, N., et al. 2017, ApJ, 845, 116

Wilson, C. D. 2018, MNRAS, 477, 2926

Yamagishi, M., Nishimura, A., Fujita, S., et al. 2018, ApJS, 235, 9

Zubovas, K., \& King, A. 2012, ApJ, 745, L34

Zubovas, K., \& King, A. R. 2014, MNRAS, 439, 400

Zubovas, K., Nayakshin, S., King, A., \& Wilkinson, M. 2013, MNRAS, 433, 3079 


\section{Appendix A: Possible northern extension of the molecular outflow}

The interferometric map of the total $\mathrm{CN}(1-0)$ line emission shown in the left panel of Fig. 1 presents an extended feature, offset to the north by $\sim 5^{\prime \prime}$ with respect to the molecular disc of Mrk 231. As noted in Sect. 1, Mrk 231 hosts multiple extranuclear shells and bubbles that are associated with feedback processes (e.g. Lípari et al. 2005, 2009; Morganti et al. 2016). However, most of the structures identified so far are located south of the nucleus, whereas this northern $\mathrm{CN}$ emission does not overlap with any other known optical or radio continuum component.

To further study the origin of this emission, we show in Fig. A.1 the $\mathrm{CO}(1-0)$ and $\mathrm{CN}(1-0)$ line spectra extracted from a 7" aperture, which samples the full extent of this northern $\mathrm{CN}$ component down to the $2 \sigma$ level. The fluxes measured by integrating the spectra are $13.0 \pm 0.8 \mathrm{Jy} \mathrm{km} \mathrm{s}^{-1}$ and $6 \pm 2 \mathrm{Jy} \mathrm{km} \mathrm{s}^{-1}$ for $\mathrm{CO}(1-0)$ and $\mathrm{CN}(1-0)$, respectively. Most of the emission is blueshifted in both tracers, with prominent broad blueshifted components. The $L_{\mathrm{CN}(1-0)}^{\prime} / L_{\mathrm{CO}(1-0)}^{\prime}$ ratio is $0.47 \pm 0.16$, which is intermediate between the values measured in the narrow core of the integrated line emission of Mrk 231 and in the broad wings (see Fig. 3).

The high $\mathrm{CN} / \mathrm{CO}$ line luminosity ratio and the broad blueshifted line profile suggest that the northern structure may trace an additional extended $(r>5 \mathrm{kpc})$ component of the molecular outflow. The nature of this emission needs to be investigated and confirmed with dedicated follow-up observations. However, the outflow hypothesis is also corroborated by the detection in molecular (Aalto et al. 2012; Cicone et al. 2012; Feruglio et al. 2015), and in neutral atomic gas tracers (Rupke \& Veilleux 2011) of high-velocity gas up to $\sim 2-3 \mathrm{kpc}$ north of the molecular disc, in a region that lies in between the newly discovered $\mathrm{CN}$-bright northern component and the wellknown kiloparsec-scale central molecular outflow.
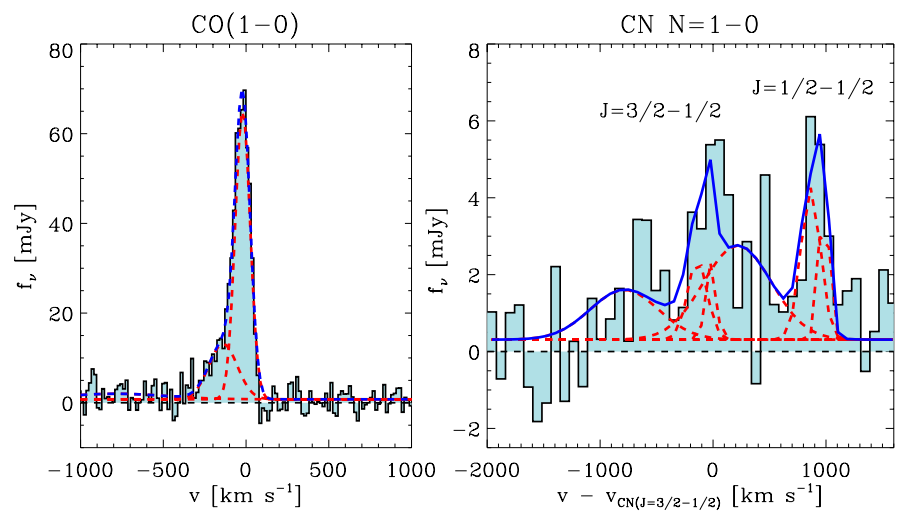

Fig. A.1. $\mathrm{CO}(1-0)$ and $\mathrm{CN}(1-0)$ emission line spectra extracted from a circular aperture with 7" radius centred at $\mathrm{RA}(\mathrm{J} 2000)=12: 56: 14.207$, $\operatorname{Dec}(\mathrm{J} 2000)=56.52 .30 .994$, sampling the northern feature of $\mathrm{CN}$ emission that is shown in the left panel of Fig. 1.

\section{Appendix B: Literature sample with $\mathrm{CN}(1-0)$ observations}

Table B.1. Literature sample.

\begin{tabular}{|c|c|c|c|c|c|c|c|c|}
\hline $\begin{array}{l}\text { Name } \\
\text { (1) }\end{array}$ & $\begin{array}{c}\mathrm{CO}(1-0) \\
(2)\end{array}$ & $\begin{array}{c}\mathrm{CN}(1-0) \\
(3)\end{array}$ & $\begin{array}{c}\mathrm{HCN}(1-0) \\
(4)\end{array}$ & $\begin{array}{c}\mathrm{HNC}(1-0) \\
(5)\end{array}$ & $\begin{array}{c}\mathrm{HCO}^{+}(1-0) \\
(6) \\
\end{array}$ & $\begin{array}{l}\text { Type } \\
(7)\end{array}$ & $\begin{array}{l}\text { Units } \\
(8)\end{array}$ & $\begin{array}{c}\text { Ref. } \\
(9)\end{array}$ \\
\hline Arp 220 & $470.7 \pm 0.9$ & $44.3 \pm 0.9$ & $54.9 \pm 1.2$ & $38.6 \pm 1.7$ & $22.9 \pm 1.0$ & I & $\mathrm{Jy} \mathrm{km} \mathrm{s}^{-1}$ & $\mathrm{a}$ \\
\hline IC 694 & 283.5 & $13 \pm 3^{(*)}$ & $11.8 \pm 1.8$ & $4.9 \pm 1.2$ & $19.2 \pm 1.9$ & I & $\mathrm{Jy} \mathrm{km} \mathrm{s}^{-1}$ & $\mathrm{~b}$ \\
\hline NGC 3690 & $290 \pm 30$ & $61 \pm 12^{(*)}$ & 14. & - & 11.1 & I & $\mathrm{Jy} \mathrm{km} \mathrm{s}^{-1}$ & $\mathrm{~b}, \mathrm{c}$ \\
\hline NGC 1614 & $201.6 \pm 0.9$ & $11.5 \pm 1.1$ & $4.7 \pm 1.7$ & $1.4 \pm 1.4$ & $9.0 \pm 1.8$ & I & $\mathrm{Jy} \mathrm{km} \mathrm{s}^{-1}$ & $\mathrm{~d}$ \\
\hline NGC 2146 & $1150 \pm 30$ & $49 \pm 12^{(*)}$ & $<18.7$ & $7.5 \pm 1.4$ & $19.7 \pm 3.3$ & I & $\mathrm{Jy} \mathrm{km} \mathrm{s}^{-1}$ & $\mathrm{~b}, \mathrm{c}$ \\
\hline NGC 2623 & 161 & 13.4 & $7.8 \pm 0.7$ & - & $4.0 \pm 0.7$ & I & $\mathrm{Jy} \mathrm{km} \mathrm{s}^{-1}$ & $e, f$ \\
\hline NGC 6240 & $327 \pm 3$ & $34.0 \pm 1.7$ & $16.7 \pm 0.9$ & $3.3 \pm 0.8$ & $27.1 \pm 1.0$ & I & $\mathrm{Jy} \mathrm{km} \mathrm{s}^{-1}$ & $\mathrm{~d}, \mathrm{~g}$ \\
\hline NGC $3256^{\mathrm{N}}$ & $1168 \pm 3$ & $280 \pm 3$ & $197 \pm 1.0$ & $110.2 \pm 0.9$ & $205.3 \pm 1.0$ & $\mathrm{~N}$ & $\mathrm{~K} \mathrm{~km} \mathrm{~s}^{-1}$ & $h, i, j$ \\
\hline NGC $3256^{\mathrm{S}}$ & $1344 \pm 3$ & $73 \pm 3$ & $57.0 \pm 1.0$ & $28.1 \pm 0.9$ & $87.9 \pm 1.0$ & $\mathrm{~N}$ & $\mathrm{~K} \mathrm{~km} \mathrm{~s}^{-1}$ & $\mathrm{~h}, \mathrm{i}, \mathrm{j}$ \\
\hline NGC $3256^{\mathrm{C}}$ & $1037 \pm 2$ & $95 \pm 3$ & $93.2 \pm 1.0$ & $389.0 \pm 0.9$ & $139.5 \pm 0.9$ & OF & $\mathrm{K} \mathrm{km} \mathrm{s}^{-1}$ & $h, i, j$ \\
\hline NGC $3256^{\mathrm{TNE}}$ & $487 \pm 2$ & $25 \pm 3$ & $37.3 \pm 1.0$ & $16.4 \pm 0.9$ & $71.9 \pm 1.0$ & $\mathrm{TT}$ & $\mathrm{K} \mathrm{km} \mathrm{s}^{-1}$ & $\mathrm{~h}, \mathrm{i}, \mathrm{j}$ \\
\hline NGC $3256^{\mathrm{TSE}}$ & $749 \pm 2$ & $25 \pm 3$ & $36.6 \pm 1.0$ & $13.8 \pm 0.9$ & $70.5 \pm 1.0$ & $\mathrm{TT}$ & $\mathrm{K} \mathrm{km} \mathrm{s}^{-1}$ & $h, i, j$ \\
\hline NGC $3256^{\mathrm{TSW}}$ & $990 \pm 2$ & $23 \pm 3$ & $51.9 \pm 1.0$ & $17.5 \pm 0.9$ & $84.5 \pm 1.0$ & $\mathrm{TT}$ & $\mathrm{K} \mathrm{km} \mathrm{s}^{-1}$ & $h, i, j$ \\
\hline NGC $3256^{\mathrm{OS}}$ & $537 \pm 3$ & $33 \pm 3$ & $40.4 \pm 1.0$ & $12.3 \pm 0.9$ & $64.6 \pm 1.0$ & OF & $\mathrm{K} \mathrm{km} \mathrm{s}^{-1}$ & $h, i, j$ \\
\hline NGC $3256^{\text {SW }}$ & $1317 \pm 2$ & $67 \pm 3$ & $76.7 \pm 1.0$ & $33.3 \pm 0.9$ & $124.1 \pm 1.0$ & $\mathrm{~N}$ & $\mathrm{~K} \mathrm{~km} \mathrm{~s}^{-1}$ & $\mathrm{~h}, \mathrm{i}, \mathrm{j}$ \\
\hline NGC 7130 & $257 \pm 14$ & $20 \pm 4^{(*)}$ & 16.5 & $10.0 \pm 1.2$ & - & I & $\mathrm{Jy} \mathrm{km} \mathrm{s}^{-1}$ & $\mathrm{~b}, \mathrm{c}$ \\
\hline NGC 1808 & $1900 \pm 140$ & $154 \pm 4^{(*)}$ & $107 \pm 5$ & $45 \pm 11$ & $59 \pm 4$ & I & $\mathrm{Jy} \mathrm{km} \mathrm{s}^{-1}$ & $\mathrm{~b}$ \\
\hline NGC 660 & $659.0 \pm 1.8$ & $64.6 \pm 1.6$ & $28.7 \pm 1.2$ & $15.0 \pm 1.2$ & $29.9 \pm 1.2$ & I & $\mathrm{Jy} \mathrm{km} \mathrm{s}^{-1}$ & $\mathrm{~d}$ \\
\hline
\end{tabular}

Notes. Column (1): source name. Columns (2-6): integrated line fluxes of the corresponding transitions. Column (7): source type. I = integrated observation; $\mathrm{N}$ = nucleus; $\mathrm{OF}=$ outflow region; $\mathrm{TT}$ = tidal tail; $\mathrm{C}=$ giant molecular cloud. Column (8): units of the fluxes reported in Cols. (2-6). $\left(^{*}\right)$ In these cases only the flux of the $\mathrm{CN}(1-0) J=3 / 2-1 / 2$ spingroup is available, and it has been multiplied by a factor of 1.5 to evaluate the total $\mathrm{CN}(1-0)$ flux.

References. (a) Aladro et al. (2015); (b) Baan et al. (2008); (c) Aalto et al. (2002); (d) Costagliola et al. (2011); (e) García-Burillo et al. (2012); (f) Lutz et al. (2020); (g) Papadopoulos et al. (2012); (h) Harada et al. (2018); (i) Sakamoto et al. (2014); (j) Harada, Sakamoto (priv. comm.); (k) Takano et al. (2019); (l) Meier et al. (2015); (m) Wilson (2018); (n) Watanabe et al. (2014); (o) Privon et al. (2015); (p) Imanishi et al. (2017); (q) Gao \& Solomon (2004); (r) Watanabe et al. (2017); (s) Pety et al. (2017); (t) Nishimura et al. (2016); (u) Kauffmann et al. (2017) and J. Kauffmann (priv. comm.). 
Table B.1. continued.

\begin{tabular}{|c|c|c|c|c|c|c|c|c|}
\hline $\begin{array}{l}\text { Name } \\
(1)\end{array}$ & $\begin{array}{c}\mathrm{CO}(1-0) \\
(2)\end{array}$ & $\begin{array}{c}\mathrm{CN}(1-0) \\
(3)\end{array}$ & $\begin{array}{c}\mathrm{HCN}(1-0) \\
(4)\end{array}$ & $\begin{array}{c}\mathrm{HNC}(1-0) \\
\quad(5)\end{array}$ & $\begin{array}{c}\mathrm{HCO}^{+}(1-0) \\
(6)\end{array}$ & $\begin{array}{l}\text { Type } \\
(7)\end{array}$ & $\begin{array}{l}\text { Units } \\
(8)\end{array}$ & $\begin{array}{c}\text { Ref. } \\
(9)\end{array}$ \\
\hline NGC $1068^{\mathrm{CND}}$ & $155.0 \pm 1.0$ & $46.1 \pm 0.5$ & $26.4 \pm 0.6$ & $5.6 \pm 0.4$ & $13.3 \pm 0.7$ & $\mathrm{~N}$ & $\mathrm{~K} \mathrm{~km} \mathrm{~s}^{-1}$ & $\mathrm{k}$ \\
\hline $05414+5840$ & 411.1 & $17.4 \pm 0 .^{(*)}$ & $15 \pm 3$ & $<3.8$ & $9 \pm 3$ & I & $\mathrm{Jy} \mathrm{km} \mathrm{s}^{-1}$ & $\mathrm{~b}$ \\
\hline $12243-0036$ & 165.4 & $14.1 \pm 2.7^{(*)}$ & $13.0 \pm 1.6$ & $13.4 \pm 1.6$ & $9.7 \pm 1.6$ & I & $\mathrm{Jy} \mathrm{km} \mathrm{s}^{-1}$ & $\mathrm{~b}$ \\
\hline M 83 & $1121 \pm 14$ & $94.1 \pm 0.7$ & $50.5 \pm 0.6$ & $20.4 \pm 0.4$ & $46.1 \pm 0.7$ & I & $\mathrm{Jy} \mathrm{km} \mathrm{s}^{-1}$ & $\mathrm{a}$ \\
\hline $15107+0724$ & 118.1 & $11.4^{(*)}$ & $13 \pm 4$ & $7 \pm 4$ & $7 \pm 2$ & I & $\mathrm{Jy} \mathrm{km} \mathrm{s}^{-1}$ & $\mathrm{~b}$ \\
\hline $17208-0014$ & 171. & 14.5 & $11.8 \pm 0.8$ & $9.1 \pm 1.0$ & $9.1 \pm 0.8$ & I & $\mathrm{Jy} \mathrm{km} \mathrm{s}^{-1}$ & $d, f$ \\
\hline NGC $253^{\text {tot }}$ & $870 . \pm 3$ & $150.0 \pm 1.4$ & $70.0 \pm 1.0$ & $30.0 \pm 1.0$ & $59.0 \pm 1.0$ & I & $\mathrm{K} \mathrm{km} \mathrm{s}^{-1}$ & $\mathrm{k}$ \\
\hline NGC $253^{\mathrm{SB}}$ & $16000 \pm 700$ & $2540 \pm 80$ & $2420 \pm 110$ & - & $2090 \pm 100$ & $\mathrm{~N}$ & $\mathrm{~K} \mathrm{~km} \mathrm{~s}^{-1}$ & 1 \\
\hline NGC $253^{\text {r1 }}$ & $720 \pm 70$ & $33 \pm 3$ & $24 \pm 2$ & - & $27 \pm 3$ & $\mathrm{OF}$ & $\mathrm{K} \mathrm{km} \mathrm{s}^{-1}$ & 1 \\
\hline NGC $253^{\text {r10 }}$ & $1920 \pm 190$ & $112 \pm 9$ & $179 \pm 18$ & - & $153 \pm 15$ & $\mathrm{OF}$ & $\mathrm{K} \mathrm{km} \mathrm{s}^{-1}$ & 1 \\
\hline NGC $253^{\mathrm{r} 2}$ & $1920 \pm 190$. & $76 \pm 6$ & $119 \pm 12$ & - & $76 \pm 8$ & $\mathrm{OF}$ & $\mathrm{K} \mathrm{km} \mathrm{s}^{-1}$ & 1 \\
\hline IC $342^{\text {tot }}$ & $160.0 \pm 0.4$ & $20.4 \pm 0.4$ & $11.60 \pm 0.10$ & $4.5 \pm 0.10$ & $8.4 \pm 0.10$ & $\mathrm{~N}$ & $\mathrm{~K} \mathrm{~km} \mathrm{~s}^{-1}$ & $\mathrm{k}$ \\
\hline M 82 & $2658 \pm 14$ & $213 \pm 2$ & $117 \pm 2$ & $52.0 \pm 1.1$ & $167 \pm 4$ & I & $\mathrm{Jy} \mathrm{km} \mathrm{s}^{-1}$ & $\mathrm{a}$ \\
\hline NGC 4945 & $9886.6 \pm 28.4$ & $1134 \pm 30^{(*)}$ & $420 \pm 8$ & $225 \pm 4$ & $450 \pm 4$ & I & $\mathrm{Jy} \mathrm{km} \mathrm{s}^{-1}$ & $\mathrm{~b}$ \\
\hline AM2055-425 & 49 & $1.5 \pm 0.3^{(*)}$ & - & - & - & I & $\mathrm{Jy} \mathrm{km} \mathrm{s}^{-1}$ & $\mathrm{~m}$ \\
\hline AM2246-490 & 34 & $2.4 \pm 0.3^{(*)}$ & - & - & - & I & $\mathrm{Jy} \mathrm{km} \mathrm{s}^{-1}$ & $\mathrm{~m}$ \\
\hline VV114 & 613 & $6.0\left(^{*}\right)$ & - & - & - & I & $\mathrm{Jy} \mathrm{km} \mathrm{s}^{-1}$ & $\mathrm{~m}$ \\
\hline AM1300-233 & 89 & $2.6 \pm 0.3^{(*)}$ & - & - & - & I & $\mathrm{Jy} \mathrm{km} \mathrm{s}^{-1}$ & $\mathrm{~m}$ \\
\hline NGC 1377 & $45 \pm 5$ & $0.48 \pm 0.12^{(*)}$ & - & - & - & I & $\mathrm{Jy} \mathrm{km} \mathrm{s}^{-1}$ & $\mathrm{~m}$ \\
\hline IC 860 & 73.1 & 4.6 & $6.7 \pm 0.7$ & $3.6 \pm 0.6$ & $4.2 \pm 0.6$ & I & $\mathrm{Jy} \mathrm{km} \mathrm{s}^{-1}$ & $\mathrm{~d}, \mathrm{f}$ \\
\hline NGC 3079 & $1090 \pm 50$ & $32 \pm 4$ & $25.0 \pm 1.3$ & $6.6 \pm 0.9$ & $28.1 \pm 1.4$ & I & $\mathrm{Jy} \mathrm{km} \mathrm{s}^{-1}$ & $\mathrm{~d}$ \\
\hline NGC 4194 & $171.3 \pm 1.0$ & $10.0 \pm 1.2$ & $2.8 \pm 0.4$ & $1.5 \pm 0.4$ & $3.7 \pm 0.4$ & I & $\mathrm{Jy} \mathrm{km} \mathrm{s}^{-1}$ & d \\
\hline NGC 7771 & $464.0 \pm 1.4$ & $23 \pm 2$ & $20.3 \pm 0.9$ & $12.0 \pm 1.0$ & $18.5 \pm 0.9$ & I & $\mathrm{Jy} \mathrm{km} \mathrm{s}^{-1}$ & $\mathrm{~d}$ \\
\hline NGC 3556 & $251.2 \pm 1.8$ & $6.0 \pm 0.8$ & $3.2 \pm 0.5$ & $1.0 \pm 0.5$ & $5.1 \pm 0.5$ & I & $\mathrm{Jy} \mathrm{km} \mathrm{s}^{-1}$ & $\mathrm{~d}$ \\
\hline NGC 7674 & $121.1 \pm 1.2$ & $9.6 \pm 1.5$ & $<1.1$ & $<1.1$ & $<1.1$ & I & $\mathrm{Jy} \mathrm{km} \mathrm{s}^{-1}$ & $\mathrm{~d}$ \\
\hline UGC 2866 & $427.8 \pm 1.7$ & $16.0 \pm 1.2$ & $8.8 \pm 0.7$ & $4.5 \pm 0.8$ & $12.9 \pm 0.7$ & I & $\mathrm{Jy} \mathrm{km} \mathrm{s}^{-1}$ & $\mathrm{~d}$ \\
\hline UGC 5101 & $96.9 \pm 1.8$ & $22.1 \pm 1.6$ & $6.6 \pm 0.9$ & $5.4 \pm 1.1$ & $2.4 \pm 1.0$ & I & $\mathrm{Jy} \mathrm{km} \mathrm{s}^{-1}$ & $\mathrm{~d}$ \\
\hline M 51 & $249 \pm 8$ & $38.0 \pm 0.4$ & $26.7 \pm 0.5$ & $8.9 \pm 0.3$ & $13.2 \pm 0.3$ & I & $\mathrm{Jy} \mathrm{km} \mathrm{s}^{-1}$ & $\mathrm{a}$ \\
\hline M 51_P1 & $68.6 \pm 0.3$ & $3.2 \pm 0.08$ & $2.74 \pm 0.09$ & $0.96 \pm 0.06$ & $2.04 \pm 0.08$ & I & $\mathrm{K} \mathrm{km} \mathrm{s}^{-1}$ & $\mathrm{n}$ \\
\hline M 51_P2 & $54.1 \pm 0.2$ & $1 . \pm 0.3$ & $1.70 \pm 0.10$ & $0.63 \pm 0.07$ & $1.38 \pm 0.09$ & I & $\mathrm{K} \mathrm{km} \mathrm{s}^{-1}$ & $\mathrm{n}$ \\
\hline NGC 7469 & $288 \pm 7$ & $29 \pm 2$ & $11.7 \pm 0.6$ & $5.4 \pm 0.6$ & $14.3 \pm 0.7$ & I & $\mathrm{Jy} \mathrm{km} \mathrm{s}^{-1}$ & $\mathrm{a}$ \\
\hline IIIZw35 & 70.6 & 8.5 & $6.5 \pm 0.9$ & - & $3.0 \pm 0.6$ & I & $\mathrm{Jy} \mathrm{km} \mathrm{s}^{-1}$ & $e, f$ \\
\hline $05189-2524$ & 25.7 & 6.7 & $4.4 \pm 0.8$ & $2.3 \pm 0.7$ & $3.3 \pm 0.8$ & I & $\mathrm{Jy} \mathrm{km} \mathrm{s}^{-1}$ & $\mathrm{f}, \mathrm{o}$ \\
\hline $09111-1007^{\mathrm{W}}$ & 51 & 6 & $3.6 \pm 0.5$ & $<1.5$ & $5.0 \pm 1.0$ & I & $\mathrm{Jy} \mathrm{km} \mathrm{s}^{-1}$ & f,o \\
\hline $10173+0828$ & 24.2 & 2 & $2.6 \pm 0.7$ & - & $1.7 \pm 0.7$ & I & $\mathrm{Jy} \mathrm{km} \mathrm{s}^{-1}$ & $e, f$ \\
\hline $12224-0624$ & 22.6 & 3.1 & $<3.3$ & $<4.0$ & $<4.0$ & I & $\mathrm{Jy} \mathrm{km} \mathrm{s}^{-1}$ & f,o \\
\hline NGC 4418 & 117 & 15.1 & $12.9 \pm 0.9$ & $6.0 \pm 0.8$ & $7.6 \pm 0.8$ & I & $\mathrm{Jy} \mathrm{km} \mathrm{s}^{-1}$ & $\mathrm{~d}, \mathrm{f}$ \\
\hline $13120-5453$ & 234 & 39.2 & - & - & - & I & $\mathrm{Jy} \mathrm{km} \mathrm{s}^{-1}$ & $\mathrm{f}$ \\
\hline $14378-3651$ & 24.7 & 3 & - & - & - & I & $\mathrm{Jy} \mathrm{km} \mathrm{s}^{-1}$ & $\mathrm{f}$ \\
\hline $20551-4250$ & 54.9 & 3 & $1.4 \pm 0.1$ & $0.37 \pm 0.04$ & $2.20 \pm 0.10$ & I & $\mathrm{Jy} \mathrm{km} \mathrm{s}^{-1}$ & $f, p$ \\
\hline NGC 7479 & 270 & $9^{(*)}$ & $<20.2$ & - & - & I & $\mathrm{Jy} \mathrm{km} \mathrm{s}^{-1}$ & $\mathrm{f}, \mathrm{q}$ \\
\hline W51GMC & $172 \pm 4$ & $16.0 \pm 1.2$ & $5.5 \pm 0.2$ & $2.4 \pm 0.3$ & $4.5 \pm 0.2$ & $\mathrm{C}$ & $\mathrm{K} \mathrm{km} \mathrm{s}^{-1}$ & $\mathrm{r}$ \\
\hline W51GMCA & $1617 \pm 11$ & $124 \pm 7$ & $62.0 \pm 1.0$ & $40 . \pm 1.0$ & $54 . \pm 2$ & $\mathrm{C}$ & $\mathrm{K} \mathrm{km} \mathrm{s}^{-1}$ & $\mathrm{r}$ \\
\hline W51GMCB & $748 \pm 6$ & $55 \pm 4$ & $30 . \pm 0.6$ & $16.3 \pm 0.6$ & $23.4 \pm 0.6$ & $\mathrm{C}$ & $\mathrm{K} \mathrm{km} \mathrm{s}^{-1}$ & $\mathrm{r}$ \\
\hline W51GMCC & $369 \pm 6$ & $23 \pm 2$ & $11.8 \pm 0.5$ & $5.6 \pm 0.6$ & $9.3 \pm 0.5$ & $\mathrm{C}$ & $\mathrm{K} \mathrm{km} \mathrm{s}^{-1}$ & $\mathrm{r}$ \\
\hline W51GMCD & $186 \pm 6$ & $16 \pm 2$ & $5.2 \pm 0.3$ & $2.2 \pm 0.3$ & $4.5 \pm 0.3$ & $\mathrm{C}$ & $\mathrm{K} \mathrm{km} \mathrm{s}^{-1}$ & $\mathrm{r}$ \\
\hline W51GMCE & $77 \pm 3$ & $13 \pm 1.4$ & $2.1 \pm 0.3$ & $0.6 \pm 0.3$ & $1.8 \pm 0.2$ & $\mathrm{C}$ & $\mathrm{K} \mathrm{km} \mathrm{s}^{-1}$ & $\mathrm{r}$ \\
\hline W51e1/e2 & $1375 \pm 6$ & $154 \pm 3$ & $77.2 \pm 0.5$ & $50.0 \pm 0.4$ & $75.2 \pm 0.5$ & $\mathrm{C}$ & $\mathrm{K} \mathrm{km} \mathrm{s}^{-1}$ & $\mathrm{r}$ \\
\hline OrionB & 60.43 & $1.16^{(*)}$ & 1.54 & 0.445 & 1.63 & $\mathrm{C}$ & $\mathrm{K} \mathrm{km} \mathrm{s}^{-1}$ & $\mathrm{~s}$ \\
\hline LMC_N79 & $16.80 \pm 0.06$ & $0.68 \pm 0.09^{(*)}$ & $1.51 \pm 0.03$ & $0.45 \pm 0.03$ & $2.797 \pm 0.019$ & $\mathrm{C}$ & $\mathrm{K} \mathrm{km} \mathrm{s}^{-1}$ & $\mathrm{t}$ \\
\hline LMC_N44C & $25.58 \pm 0.05$ & $0.51 \pm 0.05^{(*)}$ & $1.192 \pm 0.017$ & $0.383 \pm 0.013$ & $2.164 \pm 0.015$ & $\mathrm{C}$ & $\mathrm{K} \mathrm{km} \mathrm{s}^{-1}$ & $\mathrm{t}$ \\
\hline LMC_N113 & $46.96 \pm 0.07$ & $0.90 \pm 0.12^{(*)}$ & $3.15 \pm 0.05$ & $1.39 \pm 0.03$ & $4.29 \pm 0.04$ & $\mathrm{C}$ & $\mathrm{K} \mathrm{km} \mathrm{s}^{-1}$ & $\mathrm{t}$ \\
\hline OrionA & $3.0 \pm 1.2 \times 10^{4}$ & $140 \pm 600^{(*)}$ & $1000 \pm 400$ & - & - & $\mathrm{C}$ & $\mathrm{K} \mathrm{km} \mathrm{s}^{-1} \mathrm{pc}^{2}$ & $\mathrm{u}$ \\
\hline
\end{tabular}

